\title{
Why Eddy Momentum Fluxes are Concentrated in the Upper Troposphere
}

\author{
FARID AIT-CHAALAL \\ ETH Zürich, Zurich, Switzerland \\ TAPIO SCHNEIDER \\ ETH Zürich, Zurich, Switzerland, and California Institute of Technology, Pasadena, California
}

(Manuscript received 26 August 2014, in final form 28 November 2014)

\begin{abstract}
The extratropical eddy momentum flux (EMF) is controlled by generation, propagation, and dissipation of large-scale eddies and is concentrated in Earth's upper troposphere. An idealized GCM is used to investigate how this EMF structure arises. In simulations in which the poles are heated more strongly than the equator, EMF is concentrated near the surface, demonstrating that surface drag generally is not responsible for the upper-tropospheric EMF concentration. Although Earth's upper troposphere favors linear wave propagation, quasi-linear simulations in which nonlinear eddy-eddy interactions are suppressed demonstrate that this is likewise not primarily responsible for the upper-tropospheric EMF concentration. The quasi-linear simulations reveal the essential role of nonlinear eddy-eddy interactions in the surf zone in the upper troposphere, where wave activity absorption away from the baroclinic generation regions occurs through the nonlinear generation of small scales. In Earth-like atmospheres, wave activity that is generated in the lower troposphere propagates upward and then turns meridionally, eventually being absorbed nonlinearly in the upper troposphere. The level at which the wave activity begins to propagate meridionally appears to be set by the typical height reached by baroclinic eddies. This can coincide with the tropopause height but also can lie below it if convection controls the tropopause height. In the latter case, EMF is maximal well below the tropopause. The simulations suggest that EMF is concentrated in Earth's upper troposphere because typical baroclinic eddies reach the tropopause.
\end{abstract}

\section{Introduction}

Large-scale baroclinic eddies shape the general circulation of Earth's atmosphere. They are generated in midlatitudes through baroclinic instability, propagate meridionally, and dissipate near their critical lines on the flanks of the jet streams (Randel and Held 1991). Meridionally propagating eddies transport (angular) momentum toward their generation region (Held 1975, 2000). Consequently, generation of large-scale eddies in midlatitudes and dissipation at lower and higher latitudes leads to a meridional momentum flux, with convergence in midlatitudes and divergence in the subtropics and, to a lesser extent, in polar regions (Fig. 1a).

Corresponding author address: Farid Ait-Chaalal, Geological Institute, ETH, NOG 60, Sonnegstrasse 5, 8092 Zurich, Switzerland.

E-mail: farid.chaalal@erdw.ethz.ch
The eddy momentum flux (EMF) controls the structure of the mean zonal surface wind and of meridional cells. To first order in Rossby number, surface friction balances the EMF divergence averaged over an atmospheric column in the extratropics. This balance controls the strength and direction of the mean zonal surface winds. In the upper troposphere, EMF divergence is locally balanced by the Coriolis torque acting on the meridional wind, which accounts for the mass flux in the Ferrel cell in the high-latitude polar cell and, to some extent, in the tropical Hadley cell [see Schneider (2006) for a review]. Hence, the structure of the EMF is fundamental to the mean state of Earth's atmosphere.

It is well known that EMF is concentrated in the upper troposphere, just below the tropopause (Fig. 1a). Eddy kinetic energy $\left(\mathrm{EKE} \propto \overline{u^{\prime 2}}+\overline{v^{\prime 2}}\right)$ is also maximal in the upper troposphere (Fig. 1b). However, explaining the structures of EMF and EKE is not equivalent because the correlation coefficient between $u^{\prime}$ and $v^{\prime}$ varies spatially. The absolute value of the correlation coefficient 

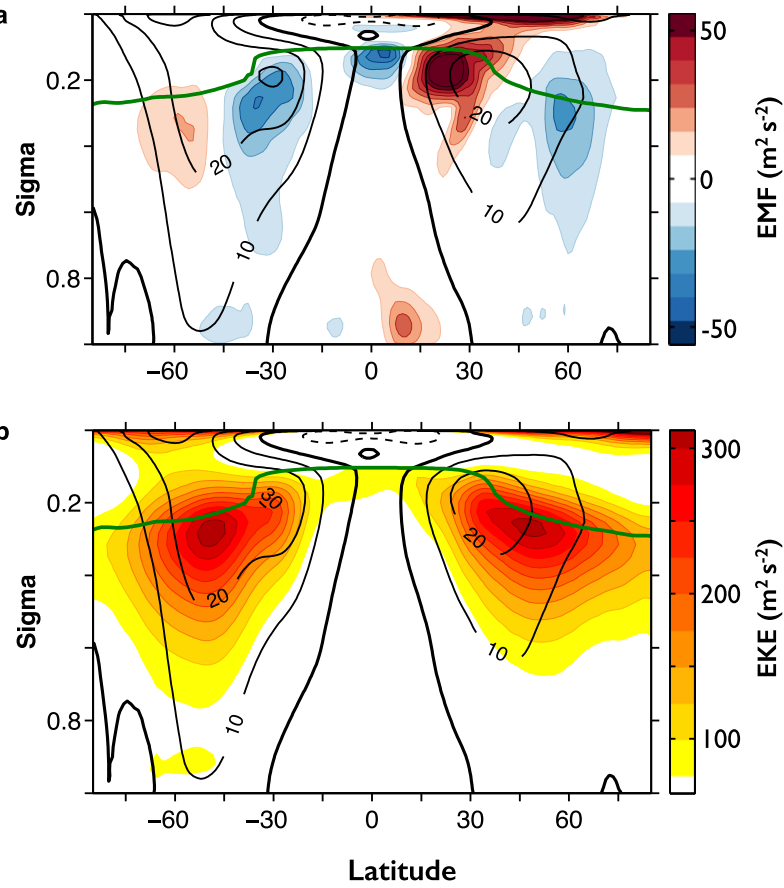

FIG. 1. Zonal wind (solid lines for eastward and dashed lines for westward; $\mathrm{m} \mathrm{s}^{-1}$ ) and (a) EMF (colors) and (b) EKE (colors). The thick green line is the tropopause (a $2 \mathrm{~K} \mathrm{~km}^{-1}$ lapse rate contour). Based on ERA-40 averaged from 1980 to 2001 (Uppala et al. 2005).

between $u^{\prime}$ and $v^{\prime}$ generally increases with height within the troposphere, from values smaller than 0.2 above the planetary boundary layer to values around 0.5 in wavebreaking regions near the tropopause. Hence, the concentration of EMF in the upper troposphere is stronger than that of EKE. The structures of EKE and EMF are not the same because EMF arises from the irreversible processes of eddy generation and dissipation, whereas EKE is nonzero even in reversible wave dynamics.

The upper-tropospheric concentration of EMF is one of the most conspicuous features of atmospheric eddy fields. Yet proposals of how it arises are scant. It has been proposed that friction acting on the eddies plays a role in reducing eddy amplitudes and meridional propagation near the surface, thus leading to reduced EMF near the surface (Held 2000; Vallis 2006). But observations of Jupiter and Saturn show that friction is unlikely to be important. On Jupiter and Saturn, EMF has been observed by tracking visible clouds in the upper troposphere, with EMF convergence in prograde (westerly) jets and divergence in retrograde (easterly) jets (Salyk et al. 2006; Del Genio et al. 2007). Although we do not have direct observations below the visible clouds, one can infer that EMF must be concentrated in a relatively shallow layer in the upper troposphere, because otherwise the implied transfer of EKE to mean-flow kinetic energy would, implausibly, exceed the total energy available to drive the flow (Schneider and Liu 2009; Liu and Schneider 2010). Indeed, simulations of Jupiter's and Saturn's atmospheres exhibit EMF concentrated in the upper troposphere, near the tropopause, much like in Earth's atmosphere, although in the simulations this is far above any drag layer, as it likely is for the actual giant planets where drag may arise magnetohydrodynamically in the planet's interior (Schneider and Liu 2009; Liu and Schneider 2010). So upper-tropospheric concentration of EMF is not unique to Earth's atmosphere but appears to be a ubiquitous feature of planetary atmospheres. And friction is unlikely to be generally responsible for the concentration, as it does not seem to play a role in giant-planet atmospheres.

Understanding the EMF concentration requires understanding the generation, propagation, and dissipation of wave activity, as pioneered in studies of baroclinic life cyles, which revealed the central role of baroclinic growth followed by barotropic decay (Simmons and Hoskins 1978, 1980; Held and Hoskins 1985; Thorncroft et al. 1993). Wave activity generation, propagation and dissipation can be diagnosed using cross sections of the Eliassen-Palm (EP) flux (Edmon et al. 1980). For dynamics that are locally quasigeostrophic (QG), the meridional and vertical components of the zonal-mean EP flux in pressure coordinates take the form

$$
\mathbf{F}=R \cos \phi\left(\frac{-\overline{u^{\prime} v^{\prime}}}{f \overline{v^{\prime} \theta^{\prime}} / \partial_{p} \bar{\theta}}\right)
$$

Here, $R$ is Earth's radius, $\phi$ is latitude, $f$ is the Coriolis parameter, $\theta$ is potential temperature, and $p$ is pressure. Primes denote departures from the zonal average $\overline{(\cdot)}$. The wave activity $A$ obeys

$$
\frac{\partial A}{\partial t}+\nabla \cdot \mathbf{F}=\mathscr{\vartheta}
$$

where 9 includes all nonconservative terms. Equation (2) has been shown to hold for small-amplitude waves, in which case the wave activity equals the pseudomomentum (Andrews and McIntyre 1976) and for finiteamplitude waves (Nakamura and Zhu 2010). When WKBJ theory is applicable, $\mathbf{F} \approx \mathbf{c}_{g} A$ is the advective flux of wave activity that is carried by the group velocity vector $\mathbf{c}_{g}$ of the waves (Lighthill and Lighthill 1960; Hayes 1977). In general, F indicates wave activity propagation. In a statistically stationary state, divergence of $\mathbf{F}$ indicates wave activity generation and convergence of $\mathbf{F}$ indicates wave activity dissipation. 
The meridional component of the EP flux is the meridional eddy angular momentum flux, which is the topic of this paper. In this sense, EMF is linked to meridional wave activity propagation. EMF divergence implies convergence of the meridional wave activity flux or wave activity dissipation. The EP flux provides a framework for connecting EMF divergence in the upper troposphere to the upward propagation of wave activity generated at lower levels.

It has been suggested that EMF is concentrated in the upper troposphere because potential vorticity (PV) gradients there are greater than in the lower troposphere, which favors meridional wave propagation (Held 2000, 2007). The larger PV gradients and stronger zonal winds aloft lead to a wider region in which Rossby wave refractive indices are positive and meridional wave propagation is possible. That is, the critical latitudes, near which meridionally propagating wave activity dissipates (Randel and Held 1991), are farther away from the wave activity generation region (Thorncroft et al. 1993). The weaker PV gradients and weaker zonal winds close to the surface, it is suggested, lead to nonlinear saturation of baroclinic eddies close to their generation latitude, precluding substantial EMF across latitudes. But the increase with height of the width of the region that allows meridional wave propagation is more gradual than the peaked structure of EMF: the meridional distance between the critical latitudes widens gradually with height, roughly like the zonal wind contours (Fig. 1a). Moreover, the zonal flow on the giant planets is thought to have an approximately barotropic structure in the upper troposphere, so that critical latitudes of waves do not vary strongly with depth. Yet EMF appears to be peaked in the upper troposphere (Liu and Schneider 2015). Such arguments based on linear wave propagation, therefore, also do not seem to account for the entire EMF structure. Even if they would, the question would remain how the mean-flow structures come to be organized in such a way that EMF becomes concentrated in the upper troposphere. The latter question also arises in the context of linear stochastic models that describe fluctuations around a prescribed mean flow (Farrell and Ioannou 1996a,b). Such models are successful in reproducing midlatitude eddy statistics, including an upper-tropospheric EMF enhancement (Whitaker and Sardeshmukh 1998; Zhang and Held 1999; DelSole 2001). But the mean flow in these models is prescribed rather than influenced by the stochastic eddies, and characteristics of the stochastic forcing are fit, for example, to observations or GCMs. So it likewise is not clear how the upper-tropospheric EMF concentration arises.
Despite their shortcomings, these appear to be the only two hypotheses that have been formulated to explain the upper-tropospheric EMF concentration: surface friction and the greater linearity of the upper troposphere. In the present paper, we test these two hypotheses explicitly with the help of an idealized dry GCM, which captures the upper-tropospheric EMF concentration and allows us to investigate it systematically. The GCM is described in section 2. Section 3 tests the friction hypothesis by discussing a circulation in which the poles are heated and the tropics are cooled, which exhibits EMF concentrated in the lower troposphere, although surface friction acts there. Section 4 tests the linearity hypothesis by comparing fully nonlinear simulations with quasi-linear (QL) simulations, in which nonlinear eddy-eddy interactions are suppressed. We show that nonlinear eddy saturation is more significant in the upper than in the lower troposphere and that eddy-eddy interactions are essential for capturing eddy absorption. In section 5, we propose a mechanism that accounts for the upper-tropospheric EMF concentration, focusing on the typical depth of baroclinic eddies, on the tropopause as a waveguide, and on nonlinear wave saturation in the upper troposphere.

\section{Idealized GCM}

The idealized dry GCM is based on the pseudospectral dynamical core of the Geophysical Fluid Dynamics Laboratory's Flexible Modeling System. It is described in detail in Schneider and Walker (2006). The only modification that we adopt is the upgrade from the Robert-Asselin filter to the Robert-Asselin-Williams filter in the leapfrog time stepping (Williams 2011). The primitive equations on a sphere are integrated using the pseudospectral method on unevenly spaced vertical $\sigma$ levels (Bourke 1974; Simmons and Burridge 1981).

The model is thermally driven by Newtonian relaxation of temperatures toward the radiative equilibrium of a semigray atmosphere. The surface temperature $T_{s}^{e}$ in radiative equilibrium is given as a function of latitude $\phi$ by

$$
T_{s}^{e}(\phi)=\tilde{T}_{s}^{e}+\Delta_{h} \cos ^{2} \phi,
$$

where $\Delta_{h}$ is the pole-to-equator temperature contrast and $\tilde{T}_{s}^{e}$ is the radiative-equilibrium surface temperature at the poles. For Earth-like simulations, we set $\Delta_{h}=90 \mathrm{~K}$ and $\tilde{T}_{s}^{e}=260 \mathrm{~K}$, with $\bar{T}_{s}^{e}=\tilde{T}_{t}^{e}+2 \Delta_{h} / 3=320 \mathrm{~K}$ the corresponding global-mean surface temperature in radiative equilibrium. The radiative-equilibrium skin temperature at the top of the atmosphere is set to $T_{t}^{e}=200 \mathrm{~K}$. The radiative-equilibrium temperature $T^{e}$ decreases 
monotonically with altitude and is given as function of the optical depth $d_{0}(\phi)$ and the pressure $p$ by

$$
T^{e}(p, \phi)=T_{s}^{t}\left[1+d_{0}(\phi)\left(\frac{p}{p_{0}}\right)^{\alpha}\right]^{1 / 4},
$$

where $\alpha=3.5$ is approximately the ratio of absorber (e.g., water vapor) scale height to density scale height. The optical depth is specified as

$$
d_{0}(\phi)=\left[\frac{T_{s}^{e}(\phi)}{T_{t}^{e}}\right]^{4}-1
$$

to achieve a continuous monotonic decrease of radiativeequilibrium temperatures from the surface to a constant temperature at the top of the atmosphere. The Newtonian relaxation time scale $\tau$ is a function of latitude $\phi$ and vertical coordinate $\sigma$, as in Held and Suarez (1994),

$$
\tau(p, \phi)=\tau_{i}^{-1}+\left(\tau_{s}^{-1}-\tau_{i}^{-1}\right) \max \left(0, \frac{\sigma-\sigma_{b}}{1-\sigma_{b}}\right) \cos ^{8}(\phi) .
$$

The constants $\tau_{i}$ and $\tau_{s}$ are the relaxation times in the interior of the atmosphere and at the surface in low latitudes. Earth-like simulations are carried out with $\sigma_{b}=0.85$, $\tau_{i}=50$ days, and $\tau_{s}=10$ days. The Newtonian relaxation scheme is presented in more detail in Schneider (2004).

The radiative-equilibrium temperature profile $T_{s}$ is statically unstable in the lower atmosphere. A convective parameterization redistributes enthalpy vertically and mimics the stabilizing effect of latent heat release in moist convection. When an atmospheric column is statically less stable than a prescribed lapse rate $\gamma \Gamma_{d}$, with dry adiabatic lapse rate $\Gamma_{d}=g / c_{p}$ and $\gamma \leq 1$, its temperature is relaxed toward $\gamma \Gamma_{d}$ on a time scale of $4 \mathrm{~h}$. Details can be found in Schneider and Walker (2006). The implied (but not explicit) latent heat release increases as $\gamma$ decreases; a value $\gamma=1$ corresponds to vertical entropy homogenization through dry convection. Earth-like simulations are performed with $\gamma=0.7$, corresponding to a lapse rate of $6.9 \mathrm{~K} \mathrm{~km}^{-1}$.

Dissipation consists of $\nabla^{8}$ hyperviscosity acting on temperature, vorticity, and divergence and of momentum and dry static energy diffusion in a $2.5-\mathrm{km}$-deep boundary layer (Smagorinsky et al. 1965).

The simulations are performed at horizontal spectral resolution T85 with $30 \sigma$ levels. All time averages are performed over 600 days after 1400 days of spinup.

\section{Heating the poles and cooling the tropics}

Latitude-dependent radiative forcing on Earth introduces a vertical asymmetry of the troposphere because, at leading order, zonal-mean vertical shear is proportional to zonal-mean meridional temperature gradients (thermal wind balance). Here we examine a circulation in which the pole-to-equator surface temperature gradient is reversed. This is achieved by setting $\Delta_{h}=-90 \mathrm{~K}$ and $\tilde{T}_{s}^{e}=380 \mathrm{~K}$; that is, radiativeequilibrium temperatures near the poles are larger than near the equator. The Newtonian relaxation time scale to this radiative-equilibrium temperature is uniform with $\tau_{s}=\tau_{i}=40$ days. All other GCM parameters are unchanged from the Earth-like simulation introduced in section 2 .

The EMF structure (Fig. 2a) is an upside-down version of the Earth-like structure (Fig. 2b). Maximal EMF occurs inside the atmospheric boundary layer, where drag is acting on the flow. Surface drag is known to affect properties of macroturbulent eddies and the general circulation, including EMF amplitude, jet strength, and jet location (James and Gray 1986; Robinson 1997; Chen et al. 2007; Liu and Schneider 2015). It has also been suggested that surface drag might at least partially explain the upper-tropospheric concentration of EMF (Held 2000; Vallis 2006). Our GCM simulation indicates that friction alone is not responsible for the uppertropospheric concentration of EMF, because the maximal convergence occurs in the frictional boundary layer when temperature gradients are reversed.

Baroclinic eddies in the simulation are generated, as on Earth, in the extratropical troposphere, as can be diagnosed from the time evolution of a simulation started from a slightly perturbed axisymmetric state with zonal winds and temperature structure equal to the zonal mean of the statistically steady state shown above. The Charney and Stern (1962) necessary condition for baroclinic instability is satisfied: although surface potential temperatures increase poleward, so that the surface temperature gradient is reversed relative to Earth's, the interior-tropospheric PV gradient is also reversed (negative) in midlatitudes. The quasigeostrophic potential vorticity (QGPV) gradient along isobars approximates the PV gradient along isentropes (Charney and Stern 1962) and is given by

$$
\partial_{y} \bar{q}=\beta-\partial_{y y} \bar{u}+f \partial_{p}\left(\frac{\partial_{y} \bar{\theta}}{\partial_{p} \bar{\theta}}\right) .
$$

The reversal of the QGPV gradient in the interior troposphere arises because the stretching term (third term on the right-hand side) dominates the planetary vorticity gradient $\beta=\partial_{y} f$, where $y=R \phi$ is the meridional distance coordinate. In other words, the slope of the isentropes, $I=-\partial_{y} \bar{\theta} / \partial_{p} \bar{\theta}$, flattens sufficiently rapidly with 


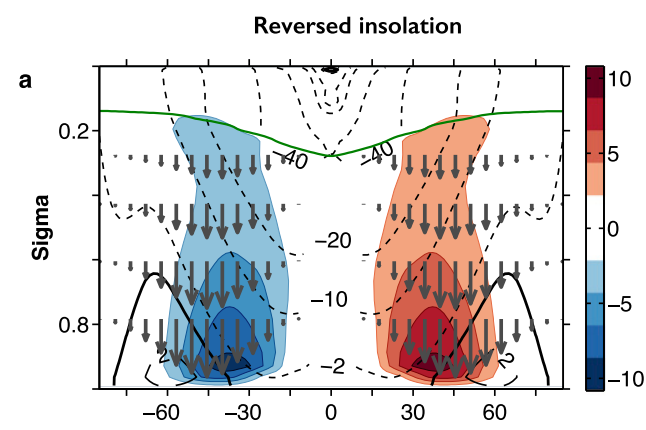

b
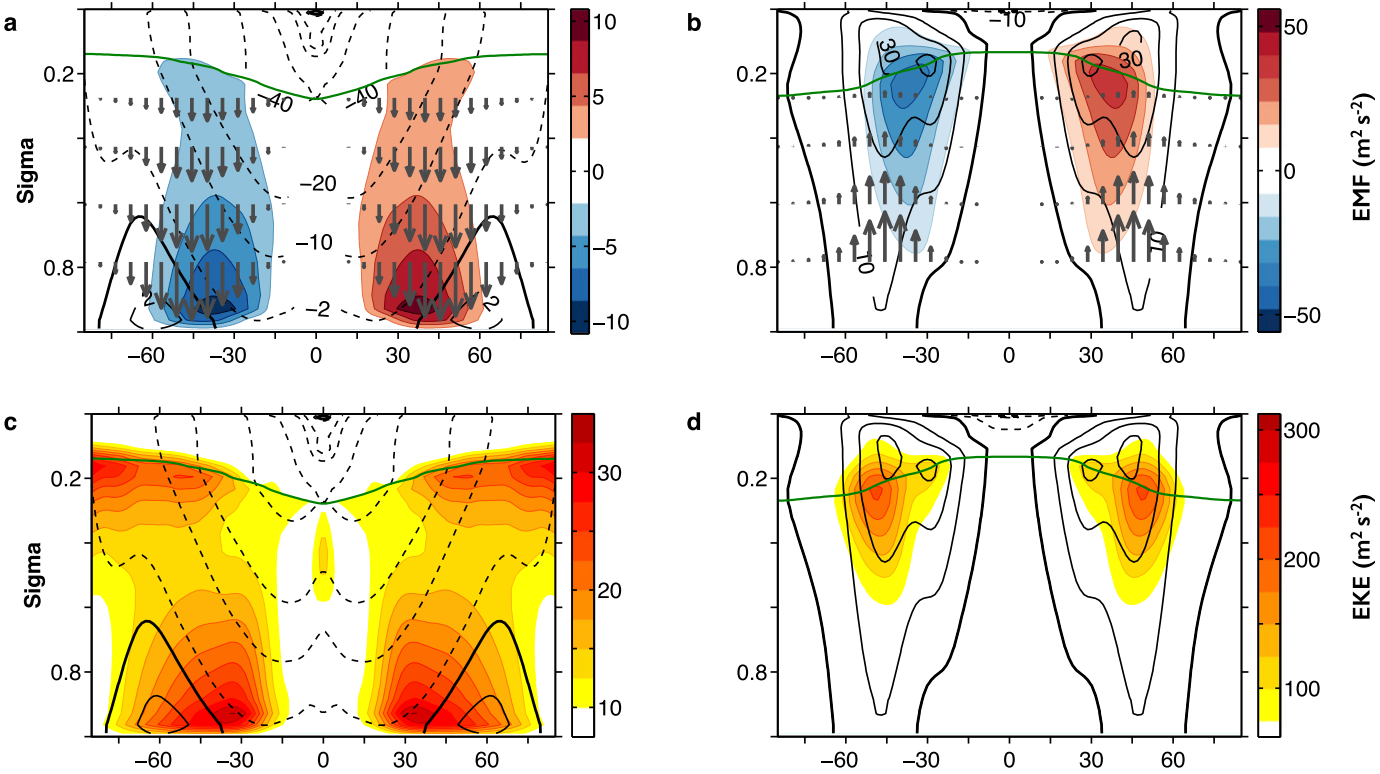

d
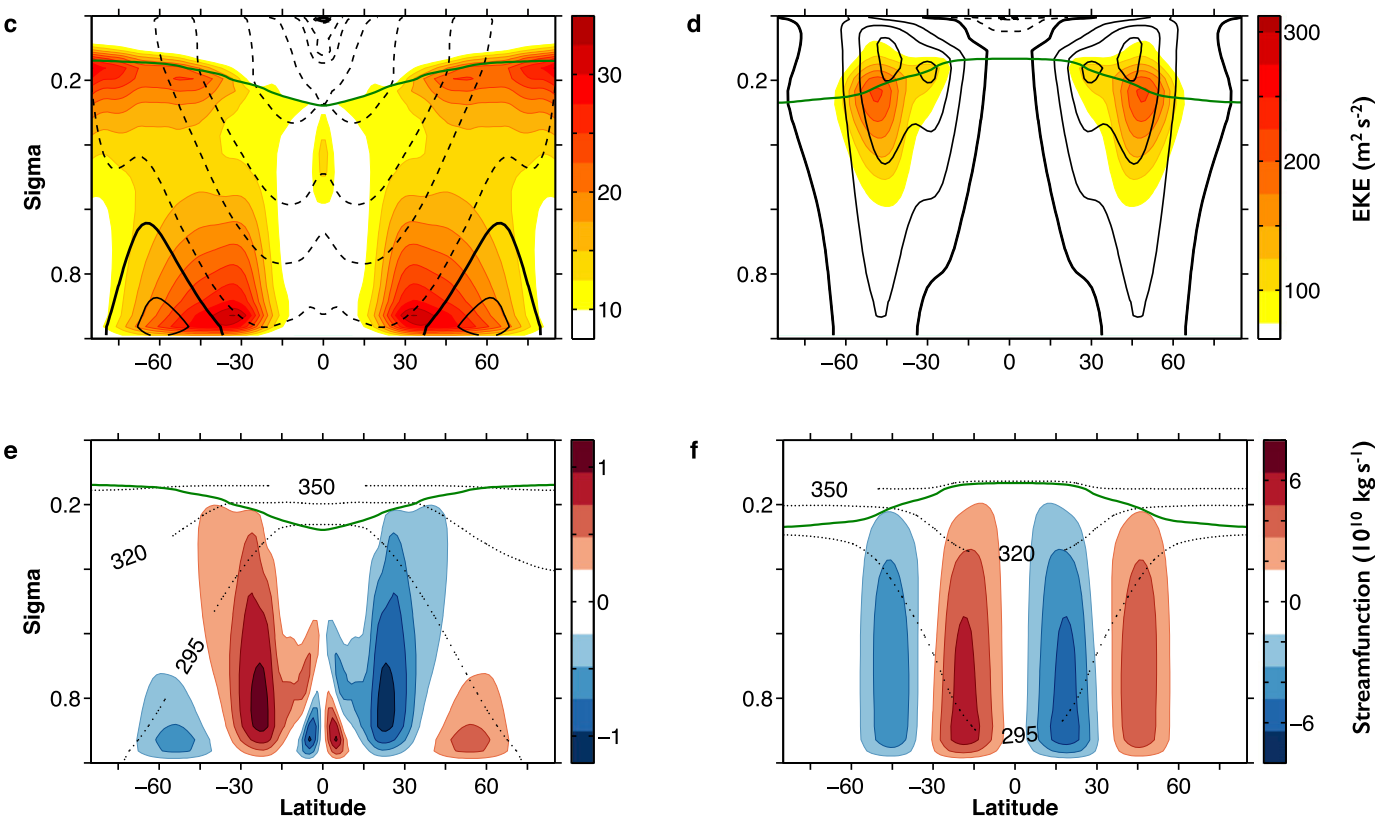

f

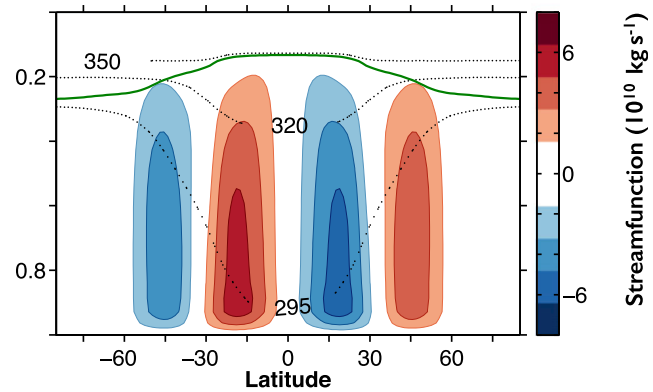

FIG. 2. Comparison of a circulation in which (a),(c),(e) the poles are heated and the tropics cooled with (b),(d),(f) an Earth-like simulation. Colors show (a),(b) EMF, (c),(d) EKE, and (e),(f) the mass streamfunction. The solid and dashed lines in (a)-(d) indicate westward and eastward zonal wind $\left(\mathrm{m} \mathrm{s}^{-1}\right)$, and the dark gray arrows the vertical component of the EP flux. Note that color scales are different in the reversed insolation and in the Earth-like configurations. The dotted lines in (e),(f) represent three isentropes $(295,320$, and $350 \mathrm{~K})$ as an indicator of the thermal structure. The thick green line marks the tropopause (a $2 \mathrm{~K} \mathrm{~km}^{-1}$ lapse rate contour). The statistically identical Northern and Southern Hemispheres are averaged, in addition to averaging zonally and temporally (over 600 days after a 1400-day spinup to reach a statistically steady state).

altitude in the interior troposphere that the stretching term reverses the sign of the QGPV gradient. In polar regions and in the subtropics, the QGPV gradient has the same sign as $\beta$, so that the Charney-Stern criterion for baroclinic instability is satisfied only in midlatitudes.

For baroclinic instability in a QG flow, the divergence of the zonal momentum flux is equal to a temporally growing exponential times a weighted vertical integral of the QGPV meridional gradient, with a strictly positive weight (Held 1975). As a consequence, linear theory points to EMF divergence where the QGPV gradient is positive throughout the column-that is, in our case, outside the baroclinic zone in midlatitudes. Angular momentum conservation then implies EMF convergence within the baroclinic zone.

Indeed, in our simulation with reversed temperature gradient, like on Earth, EMF is converging in midlatitudes and diverging at low latitudes (Fig. 2a); that is, EMF is directed poleward between the subtropical dissipation regions and the baroclinic generation region. Because of surface drag, EMF convergence in midlatitudes results in westerly surface winds, and divergence results in easterly surface winds closer to the equator. Westerlies and easterlies are associated with the analogs of Earth's Ferrel and Hadley cells, as indicated by the Eulerian streamfunction in Fig. 2e (to be 
compared with the Earth-like situation in Fig. 2f). Ferrel cells are here thermally direct but eddy driven and very shallow because EMF is concentrated close to the surface. Hadley cells are thermally indirect and also eddy driven. Close to the surface, two thermally direct and thermally driven cells can be seen equatorward of $15^{\circ}$.

Reversed meridional temperature gradients imply that eddy entropy fluxes, which are generally directed down the potential temperature gradient (e.g., Kushner and Held 1998; Held 1999) are, in the mean, equatorward. Because the vertical component of the EP flux [Eq. (1)] is proportional to the eddy potential temperature flux, it is directed downward. This is illustrated in Figs. $2 a$ and $2 b$. It implies downward, rather than the usual upward, propagation of wave activity (Edmon et al. 1980). Wave activity then accumulates near the surface in the extratropics, propagates equatorward, and dissipates, leading to EMF and EKE maxima there, despite the drag (Figs. 2a,c). Wave activity appears to propagate horizontally where farther vertical propagation is inhibited, be it by a solid boundary in the case of reversed temperature gradients or by the tropopause, an interface at which the static stability increases, in the Earth-like case.

This simulation shows that upper-level EMF enhancement for an Earth-like simulation and the underlying asymmetry between the lower and the upper parts of the troposphere cannot be attributed to surface friction alone. We have verified that this conclusion is robust when the strength of surface friction is varied. The latter has a strong effect on the near-surface zonal and eddy kinetic energies but not on the EMF strength. Similarly, we have verified that the conclusions continue to hold when surface friction is only applied to the zonal-mean flow but not to the eddies. Both in Earth-like and reversed insolation simulations, the EMF structure is qualitatively unchanged when surface friction acts only on the mean flow, which is consistent with previous studies of the separate effects of friction on mean flow and eddies (Chen et al. 2007). In both cases, we observe that EMF strength is approximately doubled when surface friction acts only on the mean flow. This likewise shows that frictional damping of eddies in Earth-like situations is not responsible for the uppertropospheric EMF enhancement.

Our reversed insolation experiment was motivated by theoretical considerations. Nevertheless, it is worth noting that it can describe the atmospheres of planets with high obliquity, which were recently studied by Ferreira et al. (2014).

\section{Quasi-linear simulations}

As explained earlier, it has been suggested that an upper troposphere that is more linear than the lower troposphere favors meridional propagation of eddies and may explain upper-tropospheric EMF enhancement (Held 2007). To test this hypothesis, we compare fully nonlinear to QL simulations for Earth-like parameters. A QL model only captures the linear behavior of eddies and their nonlinear interaction with a mean flow. Nonlinear eddy-eddy interactions are suppressed.

\section{a. Quasi-linear model}

Eddies are defined as departures from the average over longitude $\lambda$ at constant latitude $\phi$ (zonal mean):

$$
a(\phi, \lambda, p)=\bar{a}(\phi, p)+a^{\prime}(\phi, \lambda, p) .
$$

Throughout this paper, the overbar denotes a zonal average at constant pressure $p$ and the prime demotes the departure from this average. In the GCM, we use a surface pressure-weighted zonal average $\overline{p_{s}(\cdot)} / \bar{p}_{s}$ along $\sigma$ surfaces because surface pressure acts as a density in $\sigma$ coordinates (whereas the flow is nondivergent in pressure coordinates).

The QL approximation means keeping eddy-mean flow interactions and removing eddy-eddy interactions. The removal of eddy-eddy interactions in the dry GCM consists of modifying the momentum and thermodynamic equations as described by O'Gorman and Schneider (2007). For example, the time tendency owing to meridional advection of a field $a=\bar{a}+a^{\prime}$ by a velocity $v=\bar{v}+v^{\prime}$ can be decomposed as

$$
\frac{\partial a}{\partial t}=-v \frac{\partial a}{\partial y}=-\bar{v} \frac{\partial \bar{a}}{\partial y}-\bar{v} \frac{\partial a^{\prime}}{\partial y}-v^{\prime} \frac{\partial \bar{a}}{\partial y}-v^{\prime} \frac{\partial a^{\prime}}{\partial y} .
$$

The term $v^{\prime} \partial a^{\prime} / \partial y$ in Eq. (9) represents the advection of the eddies by the eddies themselves. It can be decomposed into a mean part $\left(\overline{v^{\prime} \partial a^{\prime} / \partial y}\right)$ and a fluctuating part. The removal of the eddy-eddy interactions consists of keeping only the former in Eq. (9):

$$
\frac{\partial a}{\partial t}=-v \frac{\partial a}{\partial y} \stackrel{\mathrm{QL}}{\approx}-\bar{v} \frac{\partial \bar{a}}{\partial y}-\bar{v} \frac{\partial a^{\prime}}{\partial y}-v^{\prime} \frac{\partial \bar{a}}{\partial y}-\overline{v^{\prime} \frac{\partial a^{\prime}}{\partial y}} .
$$

As a consequence, all triad interactions involving only eddy quantities are removed, such that interactions between the eddies and the mean flow are the only nonlinear interactions retained. This approach has received some attention since early studies of rotating large-scale flows. For example, small-amplitude wave activity conservation theorems have been derived within its scope (Charney and Drazin 1961; Eliassen and Palm 1961; Dickinson 1969; Boyd 1976; Andrews and McIntyre 1976, 1978). Keeping only eddy-mean flow interactions in the tendency equation [Eq. (10)] is equivalent to linearizing the equation for the fluctuating part $a^{\prime}$, keeping 
the equation for $\bar{a}$ unchanged; hence, the "quasi linear" denomination for the resulting set of equations. However, it does not imply that all nonlinearities are small because nonlinear eddy-mean flow interactions can be strong.

The QL approximation is well posed in the sense that it preserves inviscid invariants consistent with the order of the truncation: mass, angular momentum, entropy, energy, entropy variance, and, when applicable, potential enstrophy. First-order invariants are conserved because first moment equations are unchanged, and second-order invariants are conserved because neglected third-order terms only redistribute second-order inviscid invariants among scales.

In terms of a statistical closure, the moment or cumulant equations implied by the QL equations are closed at second order; third-order cumulants do not enter the second-order equations. So the QL approximation corresponds to truncating the hierarchy of cumulant equations at second order-an approximation that has recently been called CE2 and has been used to study the dynamics of barotropic jets (Marston et al. 2008; Tobias et al. 2011; Srinivasan and Young 2012; Marston et al. 2015). Statistical structural stability theory (Farrell and Ioannou 1996a,b) and some kinetic theories of statistical physics (Bouchet et al. 2013) are essentially equivalent to CE2.

\section{b. Mean zonal and meridional circulations}

O'Gorman and Schneider (2007) described the mean zonal circulation in the QL model and compared it with that in the corresponding fully nonlinear simulation. We extend here some of their findings to the EMF structure and the mean meridional circulation.

Figure 3 shows the mean zonal wind and Eulerian mean meridional streamfunction. The QL model produces upper-atmospheric jet streams above surface westerlies ("eddy-driven jets"), associated with meridional cells extending from the surface to the tropopause. The circulation in the QL approximation is compressed in the meridional direction compared with the full model. The midlatitude jet has a limited meridional extent and a secondary jet appears at higher latitudes. This is consistent with the Eulerian streamfunction, which exhibits narrower Hadley and Ferrel cells. Moreover, weaker cells appear at higher latitudes, in association with the secondary jets, such that four cells occur in each hemisphere (the contouring in Fig. 3 does not reveal the weak highlatitude cells). O'Gorman and Schneider (2007) attributed the meridional compression of the meridional circulation to the fact that the suppressed eddy-eddy interactions isotropize the eddies and thus generally increase meridional scales (Stone 1972; Shepherd 1987).

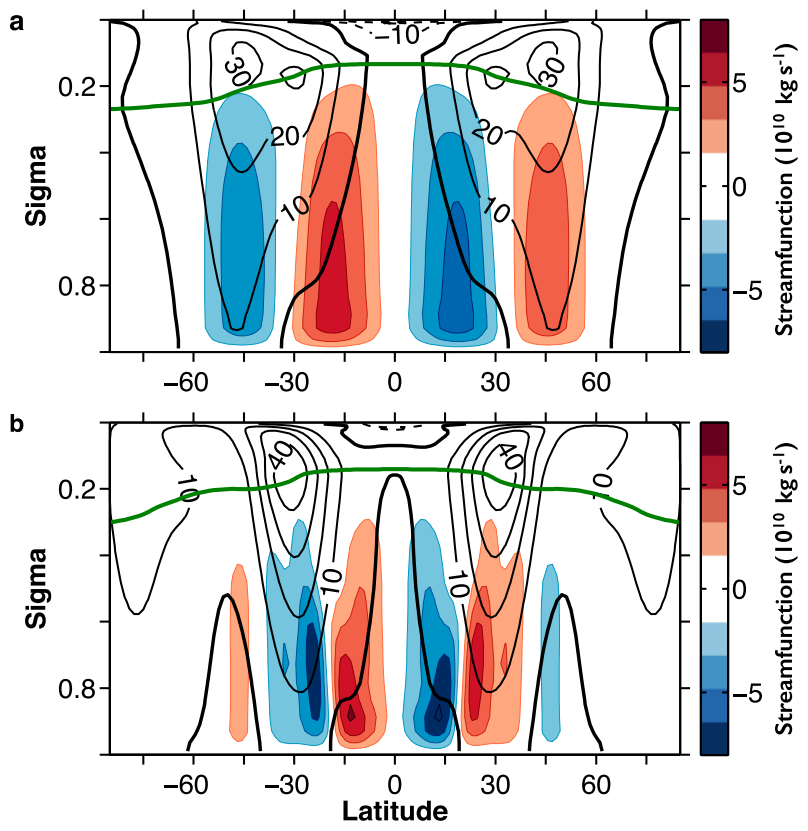

FIG. 3. Mass flux streamfunction (colors) and zonal wind $\left(\mathrm{m} \mathrm{s}^{-1}\right)$. (a) Full model and (b) QL model. The thick green line marks the tropopause. As in Fig. 2, the fields are averaged zonally, temporally, and over both hemispheres.

Thus, the QL model successfully reproduces some features of the fully nonlinear general circulation, such as eddy-driven jets and meridional cells, together with a realistic thermal structure (three isentropes are indicated in Fig. 4). However, significant qualitative and quantitative differences suggest that the redistribution of angular momentum by large-scale eddies is not realistic. We now examine the structure of the EMF.

\section{c. Eddy momentum flux}

Averages of EMF in the full model and in the QL model are compared in Fig. 4. Figure 4a depicts the wellknown picture of momentum transported by eddies in the upper troposphere from the subtropics into midlatitudes. Hence, EMF is converging above midlatitude surface westerlies and diverging above low-latitude surface easterlies. Idealized dry dynamics with a uniform surface reproduce the essence of the zonal flow and EMF structure in Earth's troposphere (Fig. 1a).

Figure $4 \mathrm{~b}$ shows that in the QL model, EMF exhibits a fundamentally different pattern. Consistent with surface westerlies around $30^{\circ}$ and $75^{\circ}$, EMF is converging in the atmospheric column above. However, instead of a poleward EMF over much of the baroclinic zone like in the full simulation, we observe in the QL simulation equatorward and poleward transport on the poleward and equatorward flanks of the jet, respectively. There 
a
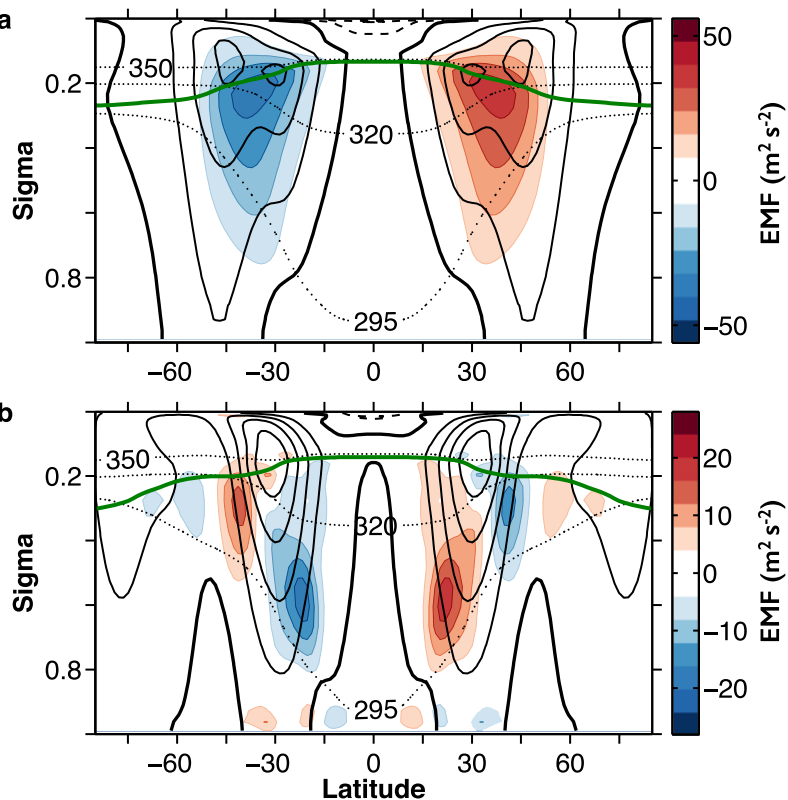

FIG. 4. EMF (colors) and zonal wind (contours; as in Fig. 3). (a) Full model and (b) QL model. Note the different color scales. The EMF amplitude in the full model is about twice as large as in the QL model. The dotted lines represent three isentropes $(295,320$, and $350 \mathrm{~K}$ ). The thick green line marks the tropopause (a $2 \mathrm{~K} \mathrm{~km}^{-1}$ lapse rate contour). As in Fig. 2, the fields are averaged zonally, temporally, and over both hemispheres.

are no well-defined upper-tropospheric extrema. Poleward EMF is maximal in the midtroposphere, which is in sharp contrast to the full simulation.

EKE, shown in Fig. 5, is maximal in the core of the jet, just below the tropopause, both in the QL and in the fully nonlinear model. Despite weak upper-tropospheric EMF, especially on the equatorward flank of the jet, EKE is still maximal near the tropopause in the QL simulation. Because the ratio of EMF to EKE can be interpreted as a correlation coefficient between meridional and zonal eddy velocity components, this implies that, contrary to the full simulation, $u^{\prime}$ and $v^{\prime}$ decorrelate in the QL upper troposphere, especially equatorward of the main midlatitude jet. Despite being of large amplitude, baroclinic eddies do not transport much angular momentum from the subtropics to midlatitudes. This suggests that the shortcomings of the QL approximation in reproducing the EMF structure do not arise because eddies would not reach the upper troposphere, for example, but because vertical propagation might not be captured adequately. Instead, the QL approximation does not capture the dissipation of eddies. To obtain a more precise picture of wave activity propagation and dissipation, we compute cross sections of the QG EP vector $\mathbf{F}$ [Eq. (1)] and of the flux of QGPV $q$, which is
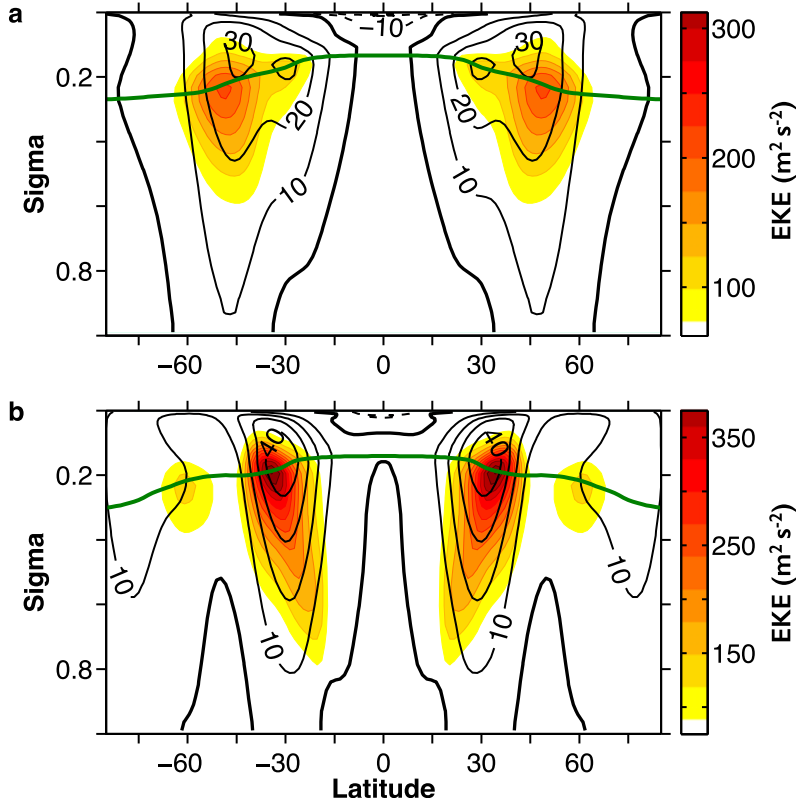

FIG. 5. EKE (colors) and zonal wind (contours; $\mathrm{m} \mathrm{s}^{-1}$ ). (a) Full model and (b) QL model. Note the different color scales. The EKE amplitude in the QL model is about twice as large as in the full model. See Fig. 4 for other details.

proportional to $\boldsymbol{\nabla} \cdot \mathbf{F}$ through the Taylor identity (Edmon et al. 1980),

$$
\overline{v^{\prime} q^{\prime}}=\frac{1}{R \cos \phi} \nabla \cdot \mathbf{F} .
$$

Both $\mathbf{F}$ and $\overline{v^{\prime} q^{\prime}}$ are shown in Fig. 6. The meridional extent of lower-tropospheric negative QGPV flux is comparable in the full and the QL models. The EP fluxes are qualitatively similar, roughly below the $295-\mathrm{K}$ isentrope, where the QGPV flux is dominated by the vertical gradient of the meridional eddy flux of potential temperature. Baroclinic growth and vertical propagation of wave activity seem fairly well captured by QL dynamics in this part of the atmosphere.

Between the 320-K isentrope and the tropopause, the EP flux is very weak in the QL model. QGPV fluxes are mostly positive, whereas in the full model, significant meridional EP flux occurs, and QGPV fluxes are negative, indicating absorption of eddies on the equatorward flank of the jet. The lack of absorption in the QL model, and even weak emission as suggested by the positive QGPV flux, accounts for the absence of enhanced EMF in the subtropical upper troposphere. It is consistent with large values of EKE being associated with weak EMF.

Interestingly, the QL model performs better in the lower troposphere than in the upper troposphere. This 
a

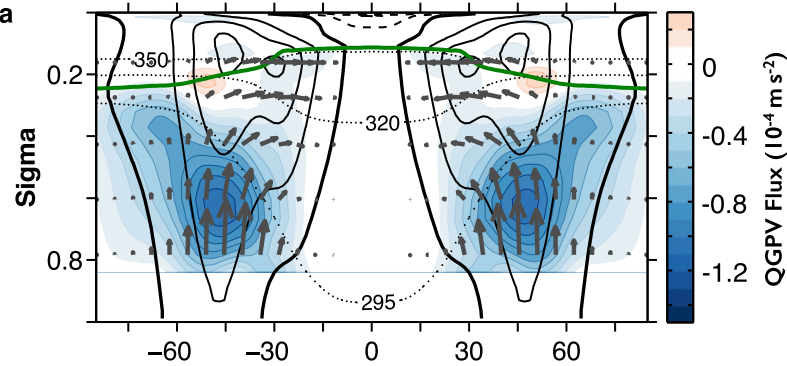

b

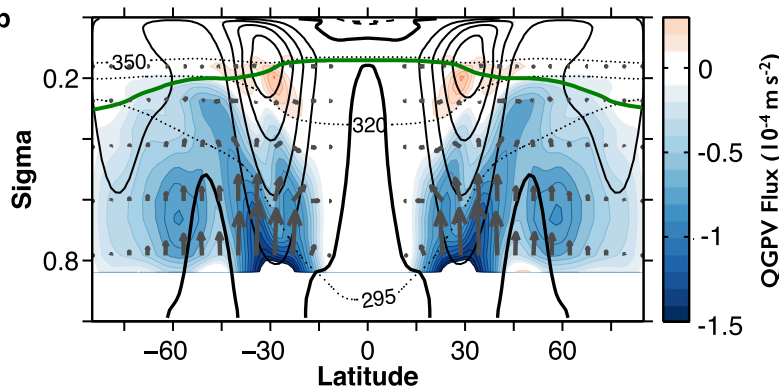

FIG. 6. Quasigeostrophic EP vector (gray arrows) and QGPV flux (colors). (a) Full model and (b) QL model. The solid and dashed contours indicate zonal wind (as in Fig. $4 ; \mathrm{m} \mathrm{s}^{-1}$ ). See Fig. 4 for other details.

appears to contradict the view of a more linear upper troposphere and of a more turbulent lower troposphere (Held 2007). However, considering time averages gives little information about the dynamical processes involved. To shed some light on the dynamics of the QL approximation and on the role of large-scale eddy-eddy interactions in eddy absorption, we perform baroclinic wave life cycle experiments.

\section{d. Life cycle experiments}

A life cycle experiment solves an initial-value problem and aims at understanding the development and saturation of a growing disturbance in a baroclinically unstable zonal flow (Simmons and Hoskins 1978, 1980; Thorncroft et al. 1993; Merlis and Schneider 2009). The initial condition that we use here is a small-amplitude disturbance of normal-mode form with respect to the mean circulation of the full model (Fig. 3). We choose the zonal wavenumber $k_{i}=6$ of the disturbance, corresponding to the fastest-growing mode for the full model. Radiative and boundary layer parameterizations are disabled. Life cycle experiments are run for the full and the QL models. In both cases, we use a normal mode of the statistically steady circulation of the fully nonlinear model.

The initial disturbance is obtained with a version of the GCM linearized around the mean circulation of the full model. We follow a similar procedure as in Merlis and Schneider (2009). As an initial condition for this linear analysis, we perturb at all vertical levels the odd

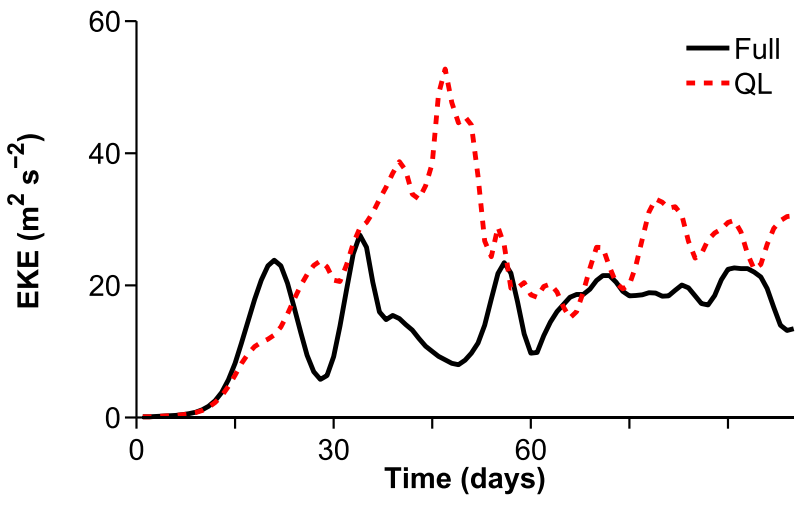

FIG. 7. Global-mean EKE as a function of time in the life cycle experiments for the full model (solid black line) and the QL model (dashed red line).

meridional spectral coefficients (from 3 to 83) of the vorticity field, corresponding to $k_{i}$. Only the spectral modes of all fields corresponding to $k_{i}$ are advanced in time. Surface pressure is uniformly rescaled such that the amplitude $\Delta p \equiv\left\langle\left(p_{s}-\bar{p}_{s}\right)^{2}\right\rangle^{1 / 2}$ is reset to $1 \mathrm{~Pa}$ when $\Delta p$ exceeds $10 \mathrm{~Pa}$ (angle brackets indicate a global average and an overbar indicates a zonal average). Temperature and velocity fields are rescaled accordingly using geostrophic balance. The rescaling procedure is repeated a few times until the disturbance growth rate and phase speed remain constant.

\section{1) ENERGy CYCLE}

Before comparing wave activity propagation in the $\mathrm{QL}$ and fully nonlinear life cycles, we briefly discuss the time evolution of EKE and of the two energy conversions involving EKE: the baroclinic conversion $C_{E}$ from eddy available potential energy (EAPE) to EKE and the barotropic conversion $C_{K}$ from zonal kinetic energy (ZKE) to EKE (Lorenz 1955). The baroclinic conversion is significant during baroclinic growth; the barotropic conversion is significant during barotropic decay, corresponding to zonal-flow acceleration through decaying eddies (Simmons and Hoskins 1978). The dominant term in $C_{K}$ involves EMF convergence (Lorenz 1955).

The evolution of EKE as a function of time is shown in Fig. 7; $C_{E}$ and $C_{K}$ are shown in Fig. 8. The fully nonlinear simulation exhibits several cycles of growth, saturation, and decay of baroclinic eddies. The two first cycles, from day 0 to day 28 and from day 28 to day 38, correspond to what is discussed in Simmons and Hoskins (1978). However, the QL model does not exhibit as clearly defined life cycles (Figs. 7 and 8b); the time evolutions of both $C_{E}$ and $C_{K}$ are different. First, there is cyclical large-amplitude conversion from ZKE to EKE, especially after day 45 (small-amplitude conversion also 

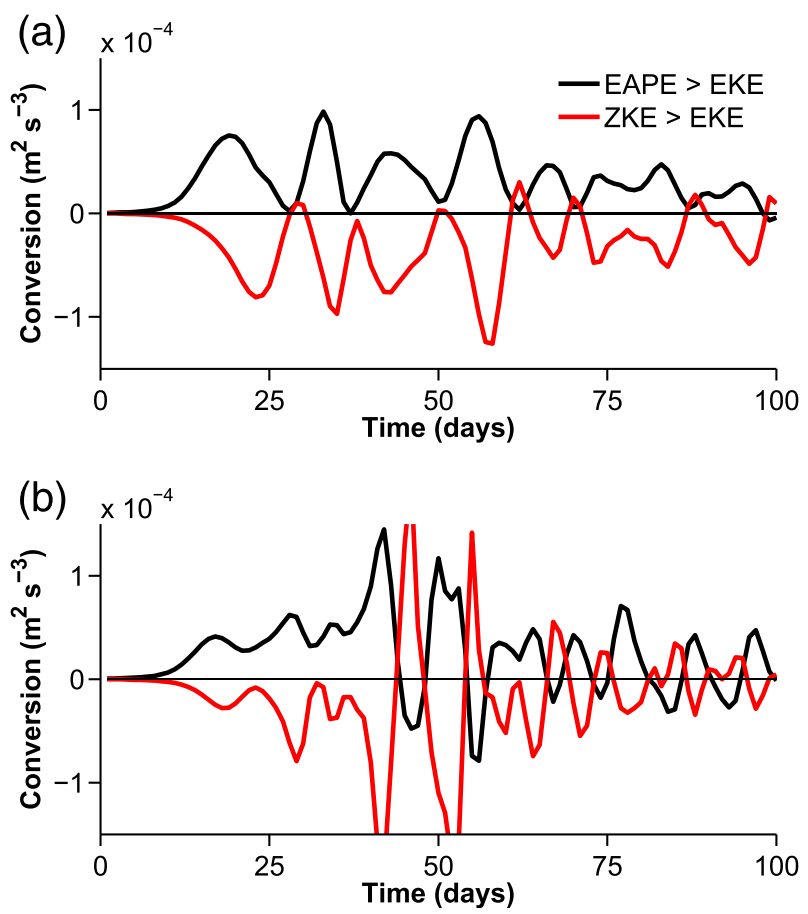

FIG. 8. Baroclinic (black) and barotropic (red) energy conversions as a function of time in the life cycle experiments for (a) the full model and (b) the QL model.

happens in the full model; for example, at day 28; see Fig. 8a). Second, when $C_{K}$ is negative, there is also relatively large conversion from EKE to EAPE, which does not occur in the full model.

We now look at wave activity propagation and absorption during barotropic decay and at the termination of life cycles to understand why QL life cycles are so different and to make the connection with the different EMF structures.

\section{2) WAVE ACTIVITY DiAgNOSTICS}

We compute cross sections of the EP flux and of the QGPV flux as in the life cycle studies of Thorncroft et al. (1993). For small-amplitude conservative eddies, the QGPV flux is proportional to the wave activity tendency, as can be seen from Eqs. (2) and (11). In addition, in the WKBJ approximation, the EP flux is transporting wave activity at the group velocity. The applicability of the small-amplitude and WKBJ approximations in the troposphere is questionable (e.g., Potter et al. 2013). Nevertheless, the QGPV flux can be interpreted as the tendency of a more general wave activity, defined for nonlinear eddies of any amplitude (Nakamura and Zhu 2010).

When the barotropic conversion of EKE to ZKE is maximal, the QL approximation captures the EP flux and wave activity tendencies fairly well (Fig. 9b). The QGPV flux in the middle to upper troposphere is negative, indicating the surf zone where eddies are absorbed. However, at the end of each life cycle, when both barotropic and baroclinic conversions are weak, there is only weak reemission of wave activity from the surf zone in the full model (Fig. 10b, left panel), while there is very strong reemission in the QL model, for example, at day 45 (Fig. 10b, right panel). Wave activity radiation from the surf zone for QL dynamics results in barotropic regrowth of eddies (positive $C_{K}$ at day 45; Fig. 9a, right panel). Associated with positive barotropic conversion is baroclinic decay (negative $C_{E}$ at day 45; Fig. 10a, right panel), which is consistent with an equatorward eddy flux of potential temperature and a downward EP flux (Fig. 10b, right panel), unlike in the full model.

The reason why wave activity on average is not absorbed in the QL upper troposphere (section 4b) can now be better understood. Low-level baroclinic growth and vertical propagation of wave activity toward the tropopause are well captured. But after wave activity is absorbed in the upper-tropospheric surf zone, it is reemitted. EP fluxes radiating from the tropical upper troposphere are particularly clear (Fig. 10b, left panel). Similar absorption followed by reemission also occurs on the poleward flank of the jet (not shown), although absorption there is of larger amplitude than emission and explains why EMF is concentrated on this flank of the jet in a statistically stationary state (Fig. 4b). Inefficient absorption of wave activity but similar baroclinicity compared with the full model explains why EKE is larger in the QL model than in the full model (section 4b): wave activity slushes meridionally in the upper troposphere, amplifying EKE and possibly leading to resonances within an upper-tropospheric waveguide.

The analysis of life cycles confirms that lowertropospheric dynamics are fairly well captured by the QL approximation and that the upper troposphere is more nonlinear than the lower troposphere (section 4b). Bursts of baroclinic growth in the lower troposphere, consisting of alternate growth and decay of EP flux, are captured. This contrasts to some extent with previous studies of baroclinic life cycles, in which low-level nonlinear saturation was invoked to explain why baroclinic conversion saturates (Simmons and Hoskins 1978; Held and Hoskins 1985; Thorncroft et al. 1993) as an essential step in a "saturation-propagation-saturation" paradigm for baroclinic wave life cycles. Our life cycle study suggests that QL mechanisms, like depletion of MAPE through baroclinic instability, might play a significant role in lower-tropospheric baroclinic growth saturation. However, strongly nonlinear mechanisms appear essential in the upper troposphere. It remains to discuss 
Full

a

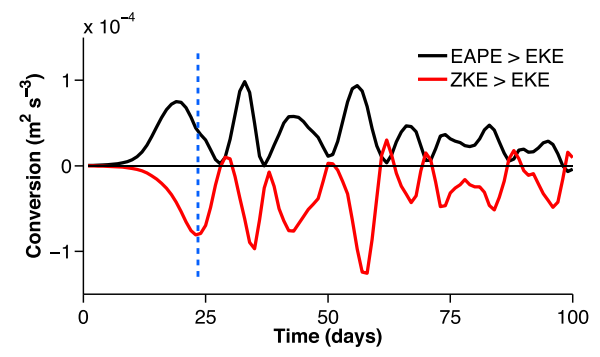

b

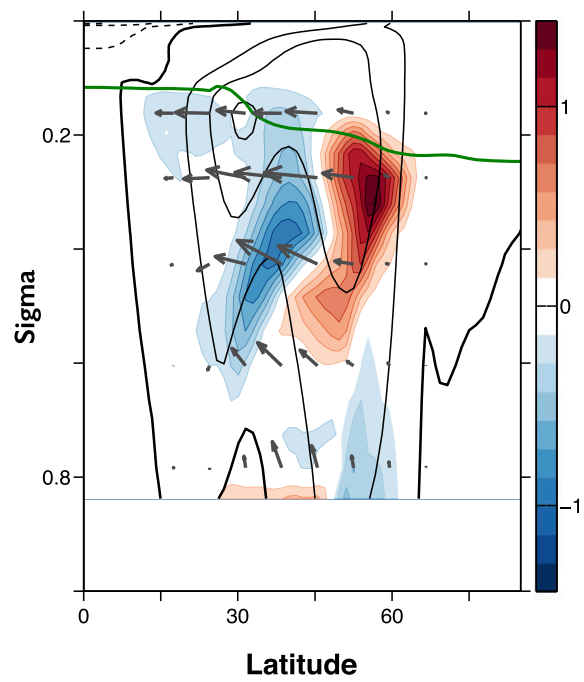

C

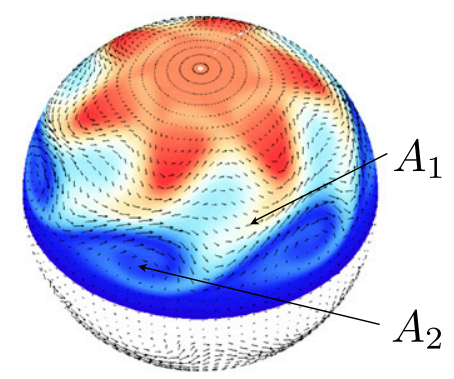

QL
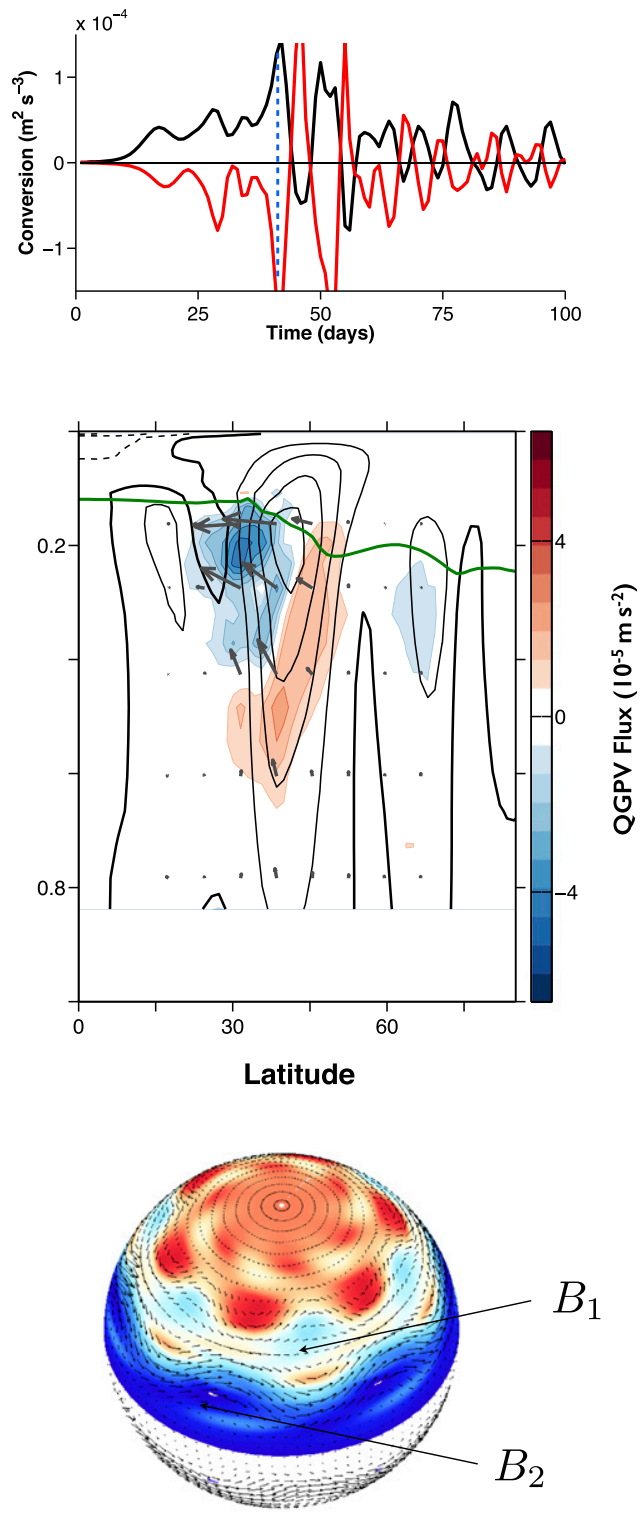

PVU

0

I

FIG. 9. EP fluxes, QGPV flux, and PV when barotropic conversion from EKE to ZKE is maximal. (left) Full model and (right) QL model. (a) A reproduction of Fig. 8 with vertical dashed blue lines added to indicate when the EP flux and the QGPV flux in (b) are computed. (b) EP flux (gray arrows) and QGPV flux (colors). The EP flux and the QGPV flux are averaged over 1 day: between days 22 and 23 for the full model and between days 28 and 29 for the QL model. As in previous figures, solid contours are for eastward winds and dashed contours for westward winds, with $10 \mathrm{~m} \mathrm{~s}^{-1}$ increments and the thick line indicating the zero contour. The green line marks the tropopause. (c) Corresponding Rossby-Ertel PV maps on the 350-K isentrope. The arrows are for the isentropicdensity-weighted winds. 
Full

a

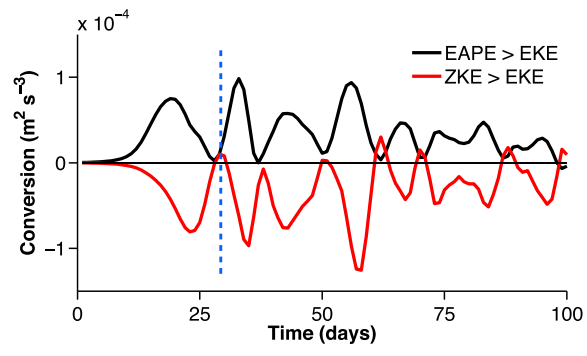

b

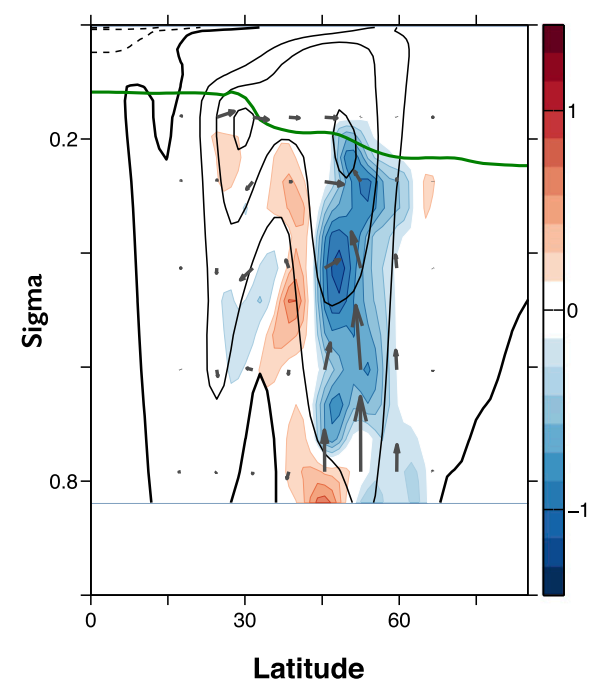

C

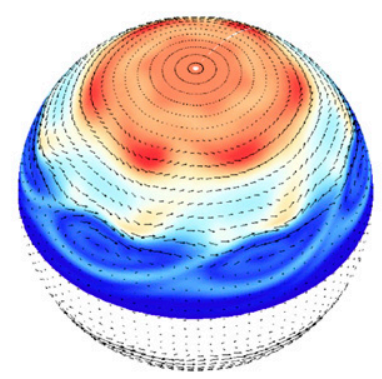

QL
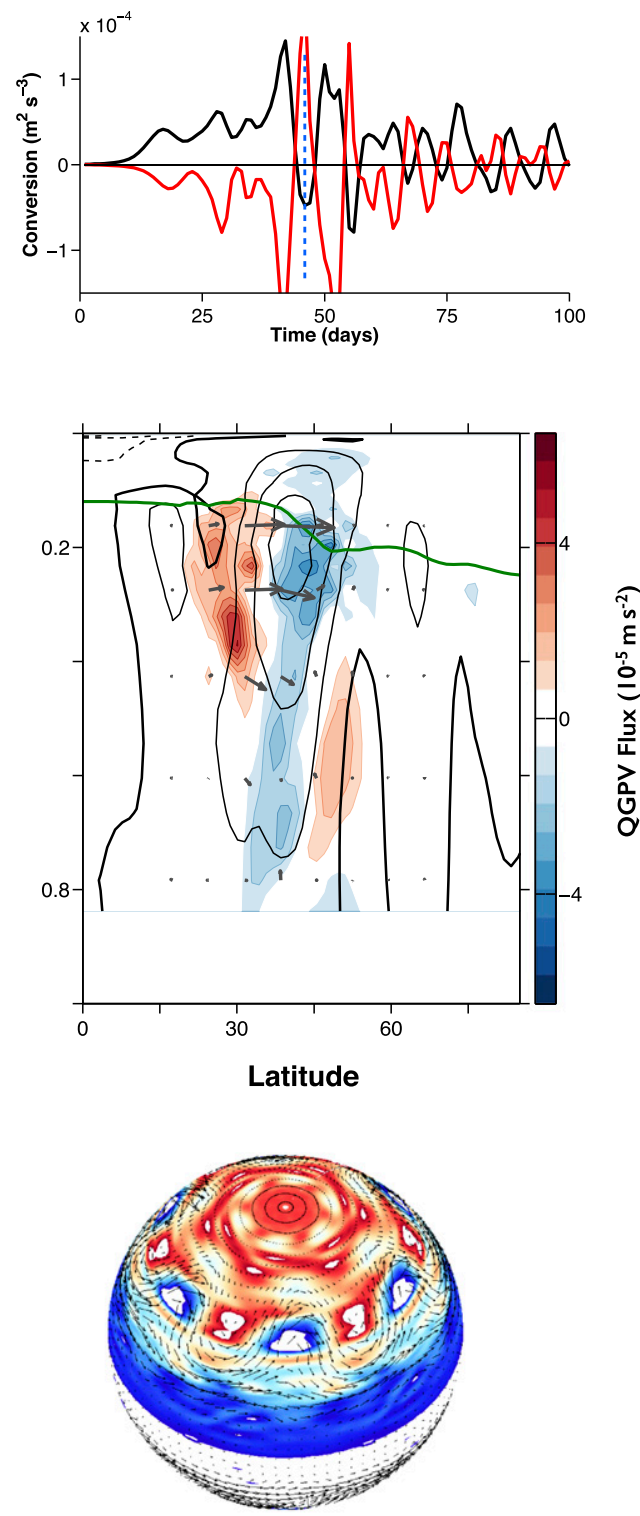

PVU

0

I

2

FIG. 10. As in Fig. 9, except at the end of a life cycle, when the barotropic conversion from EKE to ZKE is minimal.

why upper-level eddy absorption is not fully captured in the QL model.

\section{3) Potential-Vorticity REARRANGEMENT IN THE SURF ZONE}

For adiabatic inviscid motion, potential vorticity is conserved on isentropes. Analyzing potential vorticity fields on isentropes can therefore be used to diagnose wave-mean flow interactions (Hoskins et al. 1985).
Here, we use the potential vorticity for the primitiveequations system (Rossby-Ertel; PV herein) rather than the QGPV. We evaluate it on the 350-K isentrope, which lies in the upper troposphere at low latitudes and in the lower stratosphere in polar regions (Fig. 3).

In a barotropic framework, Rossby waves can be absorbed quasi linearly or nonlinearly (Held and Phillips 1987). QL absorption occurs through the Orr mechanism, when a mean flow shears eddies in the same 
direction in which they are tilted and thereby transfers their energy to the mean flow (Farrell 1987; Lindzen 1988). As the eddies are sheared, the distance between vorticity extrema decreases, and vorticity anomalies become well approximated by the ratio of velocity anomalies to typical distances between neighboring extrema. Since this distance shrinks in the shearing process, velocity anomalies, and with them EKE, have to decrease because of vorticity conservation, leading to EKE transfer to the mean flow. The nonconservation of $\mathrm{EKE}$ is a direct consequence of the linearized dynamical operator acting on eddy fields being nonnormal in the energy norm (Farrell 1987; DelSole 2004).

Eddy absorption, and the subsequent effect on the mean flow, is essentially a potential vorticity mixing problem [see Dritschel and McIntyre (2008) for a review], as expressed in the Taylor identity [Eq. (11)]. For linear decay, PV mixing is performed by the shearing in the zonal direction of meridionally propagating eddies. However, PV mixing can be nonlinear, and in general is so in planetary atmospheres. Analytical theories were developed to understand absolute vorticity (or PV) rearrangement in nonlinear Rossby wave breaking, notably the Stewartson-Warn-Warn (SWW) solution for barotropic inviscid critical layers of small-amplitude waves in a constant-shear mean flow (Stewartson 1977; Warn and Warn 1978; Killworth and McIntyre 1985). This theory predicts the formation of Kelvin cat's eye structures consisting of closed streamlines between fixed points near the critical lines, with a zonal wavenumber corresponding to the breaking wave. These structures are advecting PV anticyclonically at leading order as a passive scalar, forming small-scale PV filaments that are rolling up around the center of the cat's eye. Ultimately, this leads to vorticity mixing, which is here mediated by structures that are not zonally symmetric, which is in contrast to the linear decay.

PV maps during maximal barotropic EKE-ZKE conversion are shown in Fig. 9c. They clearly show that wave activity absorption is nonlinear in the full model. ${ }^{1}$ The point $\mathrm{A}_{1}$ marks a thinning $\mathrm{PV}$ filament, as described for LC1 wave breaking in Thorncroft et al. (1993). The region near $\mathrm{A}_{2}$ resembles the Kelvin cat's eye of a critical layer, as predicted by SWW theory. Both phenomena involve eddy-eddy interactions and enstrophy cascading toward small scales. The filament $\mathrm{A}_{1}$ constitutes the

\footnotetext{
${ }^{1}$ The increasing amplitude of PV extrema between Figs. 9 and 10 is worth noting and indicates that the numerical scheme does not conserve PV. The nonconservation is particularly striking in the QL approximation, probably because PV is not transferred as efficiently to small scales where it can be dissipated by hyperviscosity.
}

eastern flank of a structure also reminiscent of a cat's eye. Rollup of filaments is not visible on the $350-\mathrm{K}$ isentrope we are showing, but it is visible at lower levels. Corresponding structures arise in the QL model (near $\mathrm{B}_{1}$ and $\mathrm{B}_{2}$ in Fig. 9c). Barotropic linear theory of critical layers for small-amplitude waves indeed predicts the development of cat's eyes (Dickinson 1970) before linear theory breaks down. QL dynamics cannot capture the subsequent thinning and rollup of PV filaments, which are essential for vorticity mixing and the absorption of wave activity in critical layers. However, QL dynamics does capture the formation of cat's eyes (Haynes and McIntyre 1987). The baroclinic structures we observe here are more complex, but the simplified barotropic small-amplitude framework elucidates why they develop.

In contrast to the full model, in the QL model, dipoles of positive and negative $\mathrm{PV}$ anomalies near $\mathrm{B}_{1}$ and $\mathrm{B}_{2}$ in Fig. 10 persist because eddy-eddy interactions are not available to excite zonal wavenumbers larger than that of the breaking wave to allow filamentation. Haynes and McIntyre (1987) describe a similar phenomenon for the QL SWW solution. The rotation of PV anomalies around the center of the cat's eye leads to alternate phases of absorption and overreflection of the same amplitude (instead of decreasing amplitude implied by filamentation in the fully nonlinear case). Reemission of wave activity in our experiments is the baroclinic largeamplitude equivalent of the overreflection phase. When the positive vorticity anomaly is on the eastern side of the cat's eye, the tilt is southwest-northeast such that EMF is poleward. When the vorticity anomaly is advected to the west of the cat's eye, the tilt becomes southeast-northwest because it joins with the positive anomaly northwest of the cat's eye. Hence, EMF is equatorward. This is the essence of the overreflection phase. Because the phase of absorption, in which the correlation between $u^{\prime}$ and $v^{\prime}$ is positive, is followed by a phase of overreflection, in which the correlation is negative, the correlation is on average close to zero, as observed in the statistically stationary circulation (section 4c).

It is important to stress that nonlinear structures mediating PV rearrangement (cat's eyes) have a large meridional extent, spanning much of the baroclinic zone in both the full and the QL simulations. The small-amplitude calculations of Dickinson (1970) and Haynes and McIntyre (1987) are consistent with our finite-amplitude computation: similar cat's eye structures arise in both the nonlinear and the QL simulations. The fundamental difference between the two cases lies in the details of vorticity dynamics inside the cat's eyes. 
PV maps at the end of a life cycle are shown in Fig. 10c. Weak meridional gradients and smallamplitude zonal structures in the fully nonlinear simulation are a consequence of eddies having been absorbed. In the QL model, large-amplitude waves and strong PV gradients indicate wave resonance resulting from wave activity reemission. The absence of eddy-eddy interactions prevents PV filamentation and transfer of enstrophy and of wave activity toward small scales, where they can be dissipated. However, the shortcomings of the QL model are already manifest within the framework of conservative dynamics. For barotropic SWW critical layers, this is shown in Haynes and McIntyre (1987). The exact role of diffusion in wave absorption in a more complex system is not clear.

EMF is concentrated on the poleward flank of the jet in the QL simulation (Fig. 4). More work is required to understand this fact. We can conjecture that QL absorption through the Orr mechanism is more efficient on the poleward flank of the jet. Indeed, PV maps do not show the formation of cat's eye on this flank of the jet but only the shearing of eddies by the mean flow.

\section{e. The role of barotropic triads}

The life cycle calculations show that wave decay is primarily nonlinear for Earth-like parameters. To determine what components of atmospheric turbulence are crucial, we have also considered a simplified GCM in which only barotropic triads are retained, while baroclinic-baroclinic triads and baroclinic-barotropic triads are neglected. Turbulence, here taken to mean transfer of inviscid quadratic invariants among scales, is contained in the barotropic mode only. Defining a vertical average with square brackets and a zonal average with an overbar, the time tendency of a scalar $a=\bar{a}+a^{\prime}$ due to the meridional advection by the velocity $v=\bar{v}+v^{\prime}$ is integrated as follows [cf. Eq. (10)]:

$\frac{\partial a}{\partial t}=-\bar{v} \frac{\partial \bar{a}}{\partial y}-\bar{v} \frac{\partial a^{\prime}}{\partial y}-v^{\prime} \frac{\partial \bar{a}}{\partial y}-\overline{v^{\prime} \frac{\partial a^{\prime}}{\partial y}}-\left(\left[v^{\prime}\right] \frac{\partial\left[a^{\prime}\right]}{\partial y}-\overline{\left[v^{\prime}\right] \frac{\partial\left[a^{\prime}\right]}{\partial y}}\right)$.

Restoring the barotropic triads gives an EMF structure that is much closer to the full model. The uppertropospheric enhancement, with realistic amplitudes, is captured, as can be seen in Fig. 11a. This is consistent with EP vectors extending toward the subtropical upper troposphere and showing eddy absorption at the equatorward flank of the jet (Fig. 11b). Nevertheless, eddies are still compressed in the meridional direction, and secondary eddy-driven jets are developing, though at significantly higher latitudes than in the QL model.
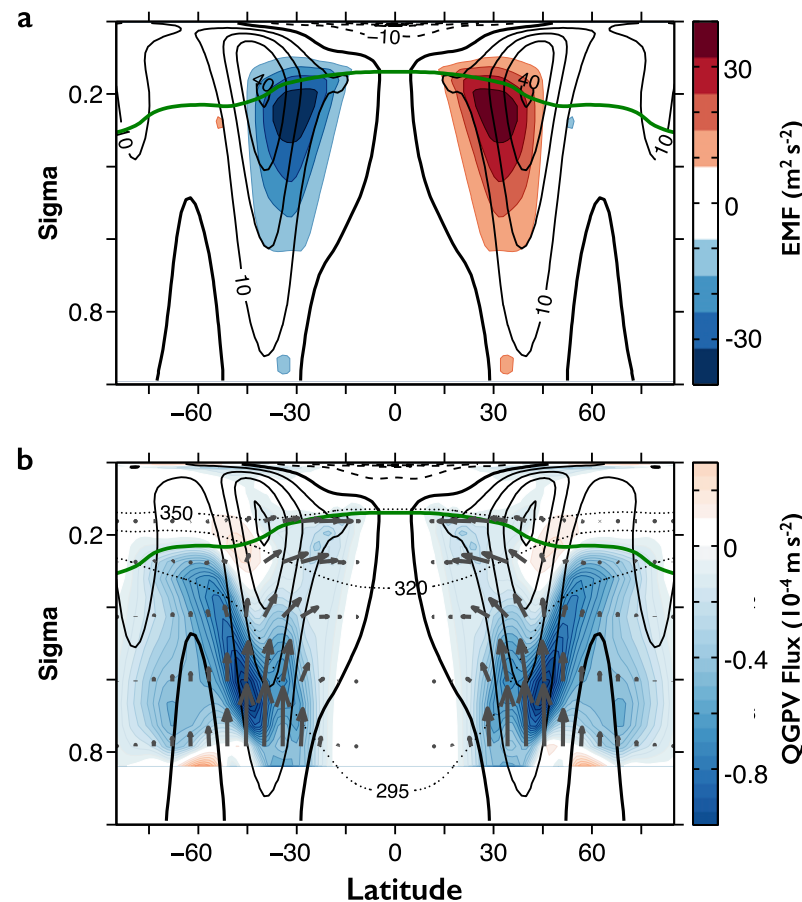

FIG. 11. Simplified GCM with wave-mean flow and barotropic eddy-eddy interactions, as described in section 4e. (a) Zonal wind and EMF (as in Fig. 4). (b) EP vector and QGPV flux (as in Fig. 6).

Eddy absorption on the poleward flank of the jet is reduced compared with the QL model but is still more significant than in the full model (not shown). Life cycle experiments confirm that nonlinear saturation does occur on the equatorward flank of the jet. Nevertheless, absorption on the equatorward flank is less efficient than in the full model. Significant wave activity is still reemitted and then absorbed on the poleward flank of the jet. As a consequence, EMF divergence is stronger than in the full model on the poleward flank of the jet. This is likely linked to the compression in the meridional direction of the circulation. Nonetheless, it is clear that allowing the nonlinear interaction of barotropic triads suffices to obtain a much more realistic baroclinic EMF structure.

It is not clear how the baroclinic waves interact with critical layers and why barotropic interactions suffice to give the baroclinic EMF structure. The baroclinic-baroclinic interactions are likely not important because vertical wavenumber 1 dominates the vertical structure of the atmosphere: baroclinic-baroclinic interactions only can affect wavenumbers larger than 2. However, baroclinicbarotropic triads are a priori important. Neglecting baroclinic-baroclinic triads and barotropic-barotropic triads, while retaining baroclinic-barotropic triads, also captures upper-level enhancement. Thus, although dissipation of wave activity is not simply related to 
the barotropic (vertically averaged) flow, retaining barotropic interactions (barotropic-barotropic triads or baroclinic-barotropic triads) suffices to capture some eddy absorption. However, it does not capture the nonlinear dynamics fully.

\section{Why eddy momentum fluxes are concentrated in the upper troposphere}

\section{a. Summary and discussion}

In section 4, we have shown that EMF is not enhanced in the upper troposphere in the QL model with Earthlike parameters because wave activity is not absorbed on the equatorward flank of the jet stream. Baroclinic eddies can be absorbed through two fundamentally different mechanisms: nonlinear saturation and the QL Orr mechanism. Life cycle experiments show that for Earth-like parameters, and for rapidly growing baroclinic eddies, nonlinear saturation is more relevant. While the Orr mechanism can be captured by QL dynamics, nonlinear saturation cannot. Essentially, eddyeddy interactions allow wave activity absorption through PV rearrangement in the surf zone and wave activity dissipation through enstrophy transfer to small scales. Without eddy-eddy interactions, wave activity is primarily reemitted from the surf zone, leading to excessive EKE in the upper troposphere.

Yet it is possible to construct circulations in which eddy amplitudes or the geometry of the mean flow favor QL wave decay over nonlinear saturation-for example, by decreasing the pole-to-equator contrast of the radiative forcing, by decreasing surface friction, or by making the planet rotate faster (not shown). Under such circumstances, the QL approximation performs better and captures upper-level EMF enhancement more accurately. In some cases, then, fundamental aspects of the tropospheric EMF vertical structure can be understood in terms of QL dynamics without having to consider nonlinear mechanisms for wave absorption. Wave activity is generated linearly in the lower troposphere and propagates vertically. Then, the tropopause acts as a turning surface for Rossby waves, trapping them in the troposphere and guiding them to propagate meridionally. This explains why no substantial EMF extends above the tropopause. In our experiment in which the poles were heated (section 3 ), waves are propagating downward, and the solid surface plays a similar role, resulting in EMF concentration near the surface.

\section{b. Depth of baroclinic eddies}

There is a vertical level below which EMF is weak (Fig. 4). The EP flux is primarily vertical in the lower troposphere because baroclinic growth dominates the dynamics. One question arises: above which altitude does the EP flux acquire a substantial meridional component? That is, at which altitude does EMF become significant? We suggest this level to be controlled by the typical vertical extent of baroclinic eddies (Held 1978; Schneider and Walker 2006).

To verify this hypothesis, we compare simulations in which the midlatitude tropopause height is set by convection with simulations in which it is set by the typical depth of baroclinic eddies (Schneider 2004; Schneider and Walker 2006). This is achieved by changing the lapse rate $\gamma g / c_{p}$ to which the convective parameterization is relaxing temperature profiles in our idealized GCM (section 2). As the convective lapse rate is reduced ( $\gamma$ gets smaller), the tropopause rises, and convection becomes increasingly important for the extratropical thermal stratification (Schneider and Walker 2006; Schneider and O'Gorman 2008; O'Gorman 2011), setting the height of the tropopause for $\gamma \lesssim 0.6$. To resolve the upper troposphere and lower stratosphere well as the tropopause height increases, we perform these simulations with $60 \sigma$ levels instead of 30 levels in the previous simulations.

The EMF for simulations with $0.4 \leq \gamma \leq 0.9$ is shown in Figs. 12a-c together with the EP vectors and the QGPV flux in dashed contours to indicate the depth of baroclinic eddies. The convective lapse rate parameter $\gamma$ has an important effect on the EMF structure: as $\gamma$ is increased and baroclinic eddies become more important in controlling the extratropical thermal stratification, EMF become more peaked in the upper troposphere.

For small convective lapse rates (e.g., $\gamma=0.4$ ), the EMF is strong over a large vertical extent and is maximal well below the tropopause (Fig. 12a). The EMF divergence (Fig. 12b) exhibits a particularly complex structure, in comparison to larger $\gamma$, because it has two distinct local maxima: in the midtroposphere $(\sigma \approx 0.5)$ and near the tropopause $(\sigma \approx 0.1)$.

EMF convergence also exhibits two corresponding maxima, but the one in the midtroposphere is weak. To elucidate how this structure arises, we show in Fig. $12 \mathrm{~g}$ the correlation coefficient between meridional and zonal velocity anomalies $\overline{u^{\prime} v^{\prime}} /\left[\overline{u^{\prime 2} v^{\prime 2}}\right]^{1 / 2}$, where the overbar here stands for a time and zonal average. It appears that the absolute value of the correlation coefficient only has one clear midlatitude maximum: near the level of the midtropospheric EMF convergencedivergence extrema. Large absolute values of the correlation coefficient indicate large EMF relative to eddy amplitude (EKE) and, thus, significant eddy absorption. Hence, comparing Figs. 12d and 12a suggests that the midtropospheric EMF convergence-divergence 

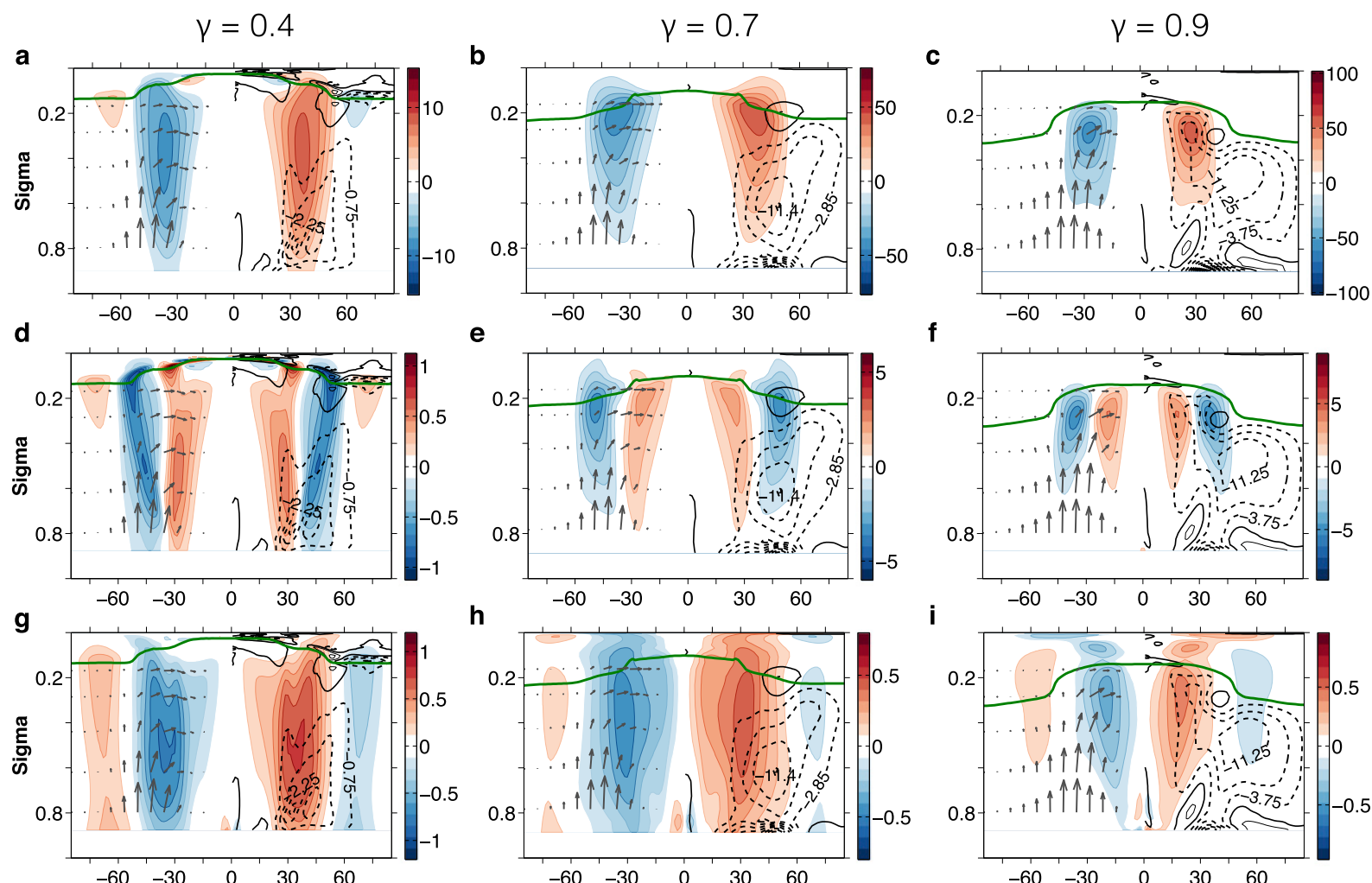

e
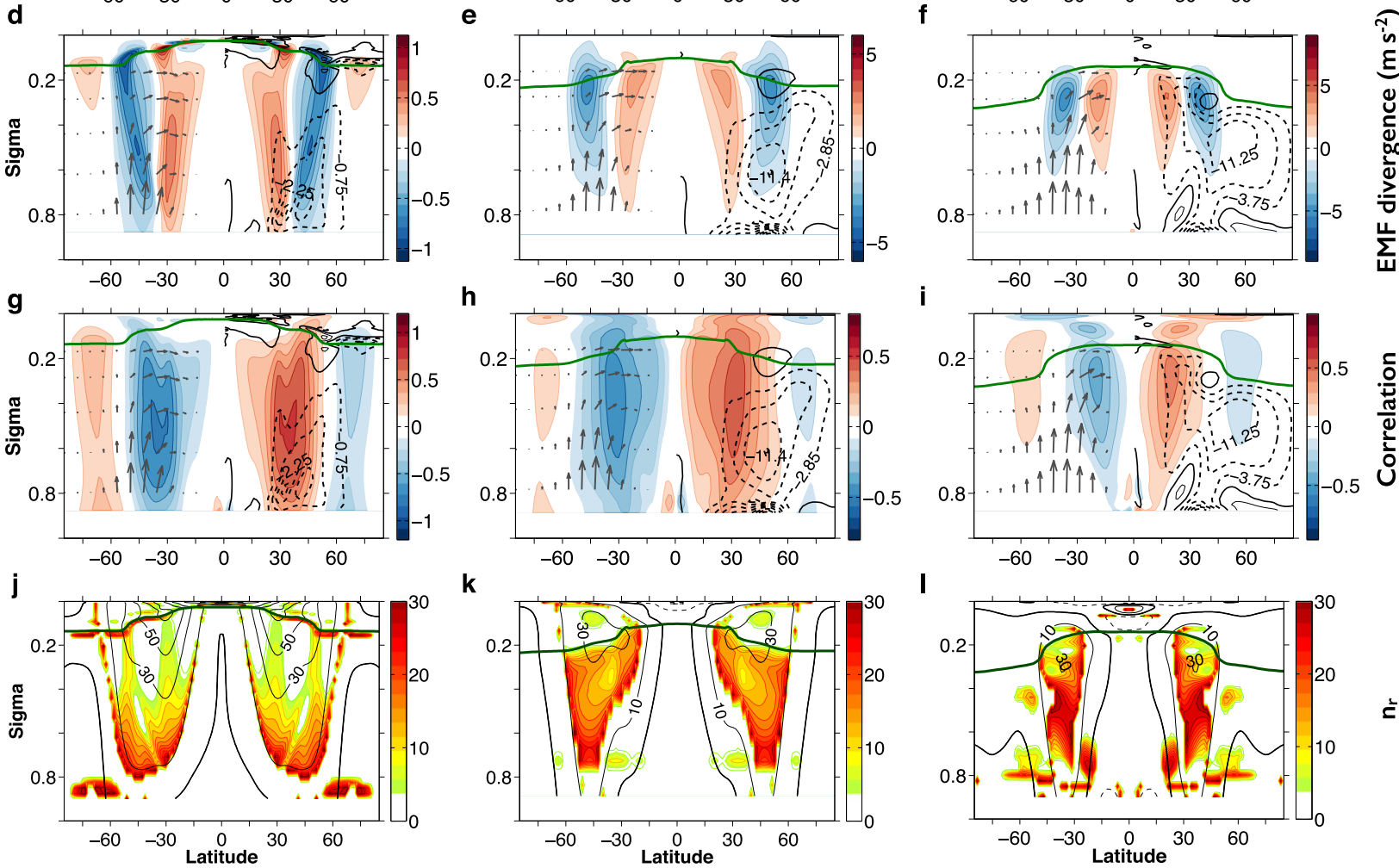

FIG. 12. (a)-(c) EMF (colors), QGPV flux (dashed contours for negative values and solid contours for positive values; $10^{-5} \mathrm{~m} \mathrm{~s}^{-2}$; Northern Hemisphere only) and the EP flux (gray arrows for midlatitudes in the Southern Hemisphere only). (d)-(f) EMF divergence (colors), QGPV flux [contours, as in (a)-(c)] and EP flux [arrows, as in (a)-(c)]. (g)-(i) Correlation $\overline{u^{\prime} v^{\prime}} /\left(\overline{u^{\prime 2} v^{\prime 2}}\right)^{1 / 2}$, QGPV flux [contours, as in (a)-(c)], and EP flux [arrows, as in (a)-(c)]. Note the change of scales for different values of $\gamma$. (j)-(l) Rossby wave refractive indices (colors) and zonal-mean wind (contours; $\mathrm{m} \mathrm{s}^{-1}$ ). In all figures, the thick green line marks the tropopause (a $2 \mathrm{~K} \mathrm{~km}^{-1}$ lapse rate contour). (left)-(right) $\gamma=0.4, \gamma=0.7$, and $\gamma=0.9$.

extrema are caused by the absorption of baroclinic eddies originating from lower levels, while the mechanisms responsible for the tropopause extrema are distinct. In fact, the EMF convergence and divergence near the tropopause correspond to northward and southward fluxes of PV (the potential temperature flux contribution to the QGPV flux there is weak, unlike in lowertropospheric layers). The northward PV flux seems upgradient, and such fluxes have recently been argued to arise from nonlinear wave breaking (Birner et al. 2013). The circulation with $\gamma=0.4$ challenges this explanation because wave-breaking regions appear well below the tropopause. Other processes may be responsible for the northward near-tropopause QGPV flux, such as local barotropic instability involving shallow modes.

As $\gamma$ increases, the altitude where the absolute value of the correlation coefficient between $u^{\prime}$ and $v^{\prime}$ in midlatitudes is maximal also increases, occurring at $\sigma=0.55,0.4$, and 0.3 for $\gamma=0.4,0.7$, and 0.9 (Figs. $12 \mathrm{~g}-\mathrm{i}$ ). This closely follows the deepening of baroclinic eddies, as shown by the tropospheric QGPV flux (e.g., Figs. 12g-i). The level up to which the EP flux penetrates and the level of maximum correlation roughly coincide. The midtropospheric maximum of EMF divergence at the vertical level of maximum correlation disappears as $\gamma$ is increased from 0.4 to 0.7 , leaving only 
the near-tropopause extrema. Apparently, the gap between the tropopause and the penetration depth of baroclinic eddies shrinks as $\gamma$ increases, both being equal for sufficiently large convective lapse rates $(\gamma \geq 0.7$; see Figs. 12a-c). But only for $\gamma=0.9$ does the vertical structure of the correlation coefficient resemble that of the EMF. The dynamics that account for the neartropopause EMF divergence-convergence extrema at $\gamma=0.4$, and contribute to it at larger $\gamma$, do not leave a noticeable signature in the correlation coefficient; they appear to be distinct from the lower-tropospheric baroclinic eddy dynamics. A simulation with $\gamma=0.4$, in which the vertical resolution is halved, accurately captures the midtropospheric EMF divergence-convergence extrema but does not exhibit the near-tropopause maxima. This also points to distinct, shallow mechanisms being responsible for the near-tropopause EMF divergenceconvergence extrema for $\gamma \leqslant 0.7$.

To diagnose where the mean flow allows Rossby wave propagation, and thus how deeply baroclinic wave can penetrate, we use refractive indices as defined in Seager et al. (2003):

$$
n_{r}^{2}=\frac{R^{2} \bar{q}_{y}}{\bar{u}-\Re(\sigma) R \cos \phi / k_{i}}-\frac{k_{i}^{2}}{\cos ^{2} \phi}+R^{2} F(N),
$$

where, in $z$ coordinates (Harnik and Lindzen 2001),

$$
F(N)=f^{2} \frac{e^{z / 2 h}}{N} \frac{\partial}{\partial z}\left[\frac{e^{-z / h}}{N^{2}} \frac{\partial}{\partial z}\left(e^{z / 2 h} N\right)\right] .
$$

We denote with $N$ and $h$ the Brunt-Väisälä frequency and the pressure scale height, respectively. The refractive index is obtained by assuming a normal-mode solution $\Psi(\phi, z) e^{i\left(k_{i} \lambda-\sigma t\right)}$ to the linearized QGPV equation around a zonal-mean flow of QGPV $\bar{q}\left(\lambda\right.$ and $k_{i}$ are the longitude and zonal wavenumber, respectively). The resulting wave equation yields (Harnik and Lindzen 2001)

$$
\nabla^{2} \Psi+n_{r}^{2} \Psi=\text { forcing and disssipation. }
$$

The vertical coordinate in the two-dimensional Laplacian operator of Eq. (15) is rescaled by the Prandtl ratio $f / N$. Positive values of $n_{r}^{2}$ indicate the possibility for wave propagation, whereas negative indices preclude it. We compute the refractive indices for the fastestgrowing normal mode on the zonal- and time-mean circulation.

Rossby wave refractive indices are consistent with baroclinic eddies not reaching the tropopause for $\gamma=0.4$ : the upper-tropospheric refractive index is negative near the jets with turning surfaces $n_{r}^{2}=0$ in the midtroposphere (Fig. 12j). For $\gamma=0.4$, baroclinic eddies are shallower than the tropopause; they do not propagate sufficiently high vertically to reach it. This is consistent with the EMF maxima matching the depth of baroclinic eddies, and it further indicates that EMF near the tropopause is due to a shallow local mechanism.

To summarize, the concentration of EMF in the upper troposphere emerges for seemingly two distinct reasons. First, baroclinic eddies appear to be absorbed at a height that is determined by their typical depth. When this depth corresponds to the tropopause height $(\gamma \gtrsim 0.7)$, EMF is enhanced in the upper troposphere. Second, near-tropopause dynamics are responsible for some meridional momentum flux (and even stronger EKE relative to lower-level baroclinic activity); the dynamical processes responsible for this are unclear but appear to be local to the tropopause.

\section{Conclusions}

Using an idealized dry GCM, we have investigated how the vertical structure of EMF is controlled and how its concentration in the upper troposphere arises. In a simulation in which the poles were heated relative to the equator, we obtained an upside-down version of Earth's tropospheric circulation with EMF and EKE enhanced close to the surface. This shows that surface friction is not responsible for Earth's weak EMF near the surface, as had been suggested (Held 2000; Vallis 2006). Nonlinear life cycle experiments are consistent with this conclusion in that they exhibit enhanced EMF in the upper troposphere, although surface friction in them is disabled.

The upper atmosphere favors linear Rossby wave propagation more than the lower troposphere. It has been suggested that this explains the EMF asymmetry between the upper and lower troposphere (Held 2007). To test this hypothesis, we compared a fully nonlinear model to a QL model, in which interactions between eddies and the mean flow are retained while nonlinear eddy-eddy interactions are suppressed. We have shown that the QL model, despite some success in capturing important aspects or planetary large-scale dynamics (O'Gorman and Schneider 2007), does not reproduce the vertical EMF structure and its concentration in the upper troposphere. The reason is that eddy absorption in Earth-like parameter regimes is strongly nonlinear: nonlinear eddy-eddy interactions in the surf zone in the upper troposphere are important for the absorption of wave activity. Wave activity is reemitted from the surf zone when eddy-eddy interactions are suppressed, as we saw in the QL baroclinic life cycle experiments. This results in excessive eddy kinetic energy in the upper 
troposphere. That is, although the upper troposphere appears relatively linear as far as wave propagation characteristics are concerned (Randel and Held 1991), it is more nonlinear than the lower troposphere with regard to eddy absorption. The QL model captures lowertropospheric dynamics (e.g., baroclinic growth and its saturation) more faithfully than upper-tropospheric dynamics (e.g., the nonlinear surf zone). The QL model does not capture the essence of the uppertropospheric dynamics because the surf zone in which nonlinear eddy-eddy interactions are important is not small in the meridional direction (as it is in some idealized flows like SWW); rather, it spans the meridional width of the baroclinic zone. An important consequence is that there is no clear separation between the latitude of generation of baroclinic eddies and the surf zone. EMF in this case occurs when dissipation occurs in the wings of eddies.

To understand the vertical EMF structure, it is fruitful to think in terms of generation, propagation, and absorption of wave activity. Wave activity is generated in the lower troposphere, propagates upward, then turns meridionally and is absorbed in the upper troposphere (Simmons and Hoskins 1978, 1980; Thorncroft et al. 1993), preferentially at a level that scales with the typical depth of baroclinic eddies. Wave activity propagation above the tropopause is inhibited because of the strong stabilization of the stratification above it, leading to the tropopause acting as a turning surface or waveguide (Thorncroft et al. 1993). As a consequence, on Earth, the EMF structure is peaked in the upper troposphere because the tropopause height and the typical depth of baroclinic eddies coincide (Held 1982; Schneider 2004; Schneider and Walker 2006; O'Gorman 2011). We have shown that when the tropopause is set by convection and baroclinic eddies do not reach the tropopause, EMF has a more complex structure with a maximum well below the tropopause that corresponds to the typical depth of baroclinic eddies. Additionally, there can be neartropopause maxima of EMF divergence-convergence generated by distinct dynamics, whose origin is not entirely clear but that also play some role in uppertropospheric EMF enhancement when the tropopause height is set by baroclinic eddies. Similarly, but with reversed sign, in the simulation in which the poles were heated more strongly than the equator, wave activity is generated in the midlatitude troposphere, propagates downward, then turns meridionally, and is absorbed near the surface. In this case, the surface plays the role of a turning surface, with EMF reaching its maximum in the lower troposphere.

A more complete understanding of the EMF structure in baroclinic atmospheres would require an explanation of why wave activity absorption occurs preferentially at a height scaling with the typical depth of baroclinic eddies. Also, near-tropopause dynamics should be investigated further because they seem to be responsible for significant EMF and so affect the large-scale circulation.

The results obtained with the QL GCM have implications for the development of statistical closures for large-scale atmospheric dynamics. What favors quasilinear or nonlinear decay, even in a simple barotropic framework, and what determines their relative importance when both can occur, is not fully understood. Unraveling this would be an essential step to develop such closures. Successes of second-order closures based on QL dynamics are likely explained by quasi-linear absorption being favored because of the structure of the flow (Farrell and Ioannou 1996a,b; Bouchet et al. 2013) or because of model tuning (Whitaker and Sardeshmukh 1998; Zhang and Held 1999; DelSole 2001). The central role of eddy-eddy interactions in planetary macroturbulence does not contradict weakly nonlinear descriptions and theories-for example, of the thermal structure (Randel and Held 1991; Held 2000; Schneider and Walker 2006, 2008)-in the sense that nonlinear eddy-eddy interactions only are essential for eddy absorption. Somewhat ironically, the atmosphere looks more linear in the presence of nonlinear eddy-eddy interactions than in the QL approximation: eddies are of weaker amplitude with respect to the mean flow in the full model than in the QL model because their absorption is inhibited in the QL model.

Acknowledgments. This work was supported by the U.S. National Science Foundation Grants CCF1048575 and CCF-1048701. We thank Brad Marston for useful discussions about quasi-linear approaches and for suggesting investigating the role of barotropic triads (section $4 \mathrm{e}$ ). We also thank Freddy Bouchet and Cesare Nardini for useful discussions about the Orr mechanism at the Kavli Institute for Theoretical Physics (KITP) summer 2014 program on wave-flow interaction in geophysics, climate, astrophysics, and plasmas.

\section{REFERENCES}

Andrews, D., and M. E. McIntyre, 1976: Planetary waves in horizontal and vertical shear: The generalized EliassenPalm relation and the mean zonal acceleration. J. Atmos. Sci., 33, 2031-2048, doi:10.1175/1520-0469(1976)033<2031: PWIHAV $>2.0 . \mathrm{CO} ; 2$.

— , and —_, 1978: Generalized Eliassen-Palm and CharneyDrazin theorems for waves in axisymmetric mean flows in compressible atmospheres. J. Atmos. Sci., 35, 175-185. 
Birner, T., D. J. Thompson, and T. Shepherd, 2013: Up-gradient eddy fluxes of potential vorticity near the subtropical jet. Geophys. Res. Lett., 40, 5988-5993, doi:10.1002/ 2013GL057728.

Bouchet, F., C. Nardini, and T. Tangarife, 2013: Kinetic theory of jet dynamics in the stochastic barotropic and 2D NavierStokes equations. J. Stat. Phys., 153, 572-625, doi:10.1007/ s10955-013-0828-3.

Bourke, W., 1974: A multi-level spectral model. I. Formulation and hemispheric integrations. Mon. Wea. Rev., 102, 687-701, doi:10.1175/1520-0493(1974)102<0687: AMLSMI > 2.0.CO;2.

Boyd, J. P., 1976: The noninteraction of waves with the zonally averaged flow on a spherical earth and the interrelationships on eddy fluxes of energy, heat and momentum. J. Atmos. Sci., 33, 2285-2291, doi:10.1175/1520-0469(1976)033<2285: TNOWWT $>2.0 . \mathrm{CO} ; 2$.

Charney, J. G., and P. Drazin, 1961: Propagation of planetary-scale disturbances from the lower into the upper atmosphere. J. Geophys. Res., 66, 83-109, doi:10.1029/JZ066i001p00083.

_ , and M. E. Stern, 1962: On the stability of internal baroclinic jets in a rotating atmosphere. J. Atmos. Sci., 19, 159-172, doi:10.1175/1520-0469(1962)019<0159:OTSOIB >2.0.CO;2.

Chen, G., I. M. Held, and W. A. Robinson, 2007: Sensitivity of the latitude of the surface westerlies to surface friction. J. Atmos. Sci., 64, 2899-2915, doi:10.1175/JAS3995.1.

Del Genio, A. D., J. M. Barbara, J. Ferrier, A. P. Ingersoll, R. A. West, A. R. Vasavada, J. Spitale, and C. C. Porco, 2007: Saturn eddy momentum fluxes and convection: First estimates from Cassini images. Icarus, 189, 479-492, doi:10.1016/ j.icarus.2007.02.013.

DelSole, T., 2001: A simple model for transient eddy momentum fluxes in the upper troposphere. J. Atmos. Sci., 58, 3019-3035, doi:10.1175/1520-0469(2001)058<3019: ASMFTE $>2.0 . \mathrm{CO} ; 2$.

_ 2004: Stochastic models of quasigeostrophic turbulence. Surv. Geophys., 25, 107-149, doi:10.1023/B: GEOP.0000028164.58516.b2.

Dickinson, R. E., 1969: Theory of planetary wave-zonal flow interaction. J. Atmos. Sci., 26, 73-81, doi:10.1175/ 1520-0469(1969)026<0073:TOPWZF>2.0.CO;2.

_ 1970: Development of a Rossby wave critical level. J. Atmos. Sci., 27, 627-633, doi:10.1175/1520-0469(1970)027<0627: DOARWC $>2.0 . \mathrm{CO} ; 2$.

Dritschel, D., and M. McIntyre, 2008: Multiple jets as PV staircases: The Phillips effect and the resilience of eddy-transport barriers. J. Atmos. Sci., 65, 855-874, doi:10.1175/ 2007JAS2227.1.

Edmon, H. J., Jr., B. J. Hoskins, and M. E. McIntyre, 1980: Eliassen-Palm cross sections for the troposphere. J. Atmos. Sci., 37, 2600-2616, doi:10.1175/1520-0469(1980)037<2600: EPCSFT $>2.0 . \mathrm{CO} ; 2$.

Eliassen, A., and E. Palm, 1961: On the transfer of energy in stationary mountain waves. Geofys. Publ., 22, 1-23.

Farrell, B., 1987: Developing disturbances in shear. J. Atmos. Sci., 44, 2191-2199, doi:10.1175/1520-0469(1987)044<2191: DDIS $>2.0 . \mathrm{CO} ; 2$.

— Part I: Autonomous operators. J. Atmos. Sci., 53, 2025-2040, doi:10.1175/1520-0469(1996)053<2025:GSTPIA >2.0.CO;2.

$\longrightarrow$, and,$- 1996 \mathrm{~b}$ : Generalized stability theory. Part II: Nonautonomous operators. J. Atmos. Sci., 53, 2041-2053, doi:10.1175/ 1520-0469(1996)053<2041:GSTPIN>2.0.CO;2.
Ferreira, D., J. Marshall, P. A. O. Gorman, and S. Seager, 2014: Climate at high-obliquity. Icarus, 243, 236-248, doi:10.1016/ j.icarus.2014.09.015.

Harnik, N., and R. S. Lindzen, 2001: The effect of reflecting surfaces on the vertical structure and variability of stratospheric planetary waves. J. Atmos. Sci., 58, 2872-2894, doi:10.1175/ 1520-0469(2001)058<2872:TEORSO > 2.0.CO;2.

Hayes, M., 1977: A note on group velocity. Proc. Roy. Soc. London, 354A, 533-535.

Haynes, P. H., and M. E. McIntyre, 1987: On the representation of Rossby wave critical layers and wave breaking in zonally truncated models. J. Atmos. Sci., 44, 2359-2382, doi:10.1175/ 1520-0469(1987)044<2359:OTRORW>2.0.CO;2.

Held, I. M., 1975: Momentum transport by quasi-geostrophic eddies. J. Atmos. Sci., 32, 1494-1497, doi:10.1175/1520-0469(1975)032<1494: MTBQGE $>2.0 . \mathrm{CO} ; 2$.

1978: The vertical scale of an unstable baroclinic wave and its importance for eddy heat flux parameterizations. J. Atmos. Sci., 35, 572-576, doi:10.1175/1520-0469(1978)035<0572: TVSOAU $>2.0 . \mathrm{CO} ; 2$.

,1982: On the height of the tropopause and the static stability of the troposphere. J. Atmos. Sci., 39, 412-417, doi:10.1175/ 1520-0469(1982)039<0412:OTHOTT $>2.0 . \mathrm{CO} ; 2$.

1999: The macroturbulence of the troposphere. Tellus, 51B, 59-70, doi:10.1034/j.1600-0889.1999.00006.x.

- 2000: The general circulation of the atmosphere. Woods Hole Lecture Notes, $70 \mathrm{pp}$. [Available online at http://www.gfdl. noaa.gov/cms-filesystem-action/user_files/ih/lectures/woods_ hole.pdf.]

2007: Progress and problems in large-scale atmospheric dynamics. The Global Circulation of the Atmosphere, T. Schneider and A. Sobel, Eds., Princeton University Press, 1-21.

—, and B. J. Hoskins, 1985: Large-scale eddies and the general circulation of the troposphere. Advances in Geophysics, Vol. 28, Academic Press, 3-31, doi:10.1016/ S0065-2687(08)60218-6.

— , and P. J. Phillips, 1987: Linear and nonlinear barotropic decay on the sphere. J. Atmos. Sci., 44, 200-207, doi:10.1175/ 1520-0469(1987)044<0200:LANBDO > 2.0.CO;2.

—, and M. J. Suarez, 1994: A proposal for the intercomparison of the dynamical cores of atmospheric general circulation models. Bull. Amer. Meteor. Soc., 75, 1825-1830, doi:10.1175/1520-0477(1994)075<1825: APFTIO $>2.0 . \mathrm{CO} ; 2$.

Hoskins, B. J., M. E. McIntyre, and A. W. Robertson, 1985: On the use and significance of isentropic potential vorticity maps. Quart. J. Roy. Meteor. Soc., 111, 877-946, doi:10.1002/ qj.49711147002.

James, I., and L. Gray, 1986: Concerning the effect of surface drag on the circulation of a baroclinic planetary atmosphere. Quart. J. Roy. Meteor. Soc., 112, 1231-1250, doi:10.1002/ qj. 49711247417.

Killworth, P. R. D., and M. E. McIntyre, 1985: Do Rossby-wave critical layers absorb, reflect, or over-reflect? J. Fluid Mech., 161, 449-492, doi:10.1017/S0022112085003019.

Kushner, P. J., and I. M. Held, 1998: A test, using atmospheric data, of a method for estimating oceanic eddy diffusivity. Geophys. Res. Lett., 25, 4213-4216, doi:10.1029/1998GL900142.

Lighthill, M. J., and M. Lighthill, 1960: Studies on magnetohydrodynamic waves and other anisotropic wave motions. Philos. Trans. Roy. Soc. London, A252, 397-430, doi:10.1098/ rsta.1960.0010. 
Lindzen, R. S., 1988: Instability of plane parallel shear flow (toward a mechanistic picture of how it works). Pure Appl. Geophys., 126, 103-121, doi:10.1007/BF00876917.

Liu, J., and T. Schneider, 2010: Mechanisms of jet formation on the giant planets. J. Atmos. Sci., 67, 3652-3672, doi:10.1175/ 2010JAS3492.1.

$\longrightarrow$, and — 2015: Scaling of off-equatorial jets in giant planet atmospheres. J. Atmos. Sci., 72, 389-408, doi:10.1175/ JAS-D-13-0391.1.

Lorenz, E. N., 1955: Available potential energy and the maintenance of the general circulation. Tellus, 7, 157-167, doi:10.1111/ j.2153-3490.1955.tb01148.x.

Marston, J. B., E. Conover, and T. Schneider, 2008: Statistics of an unstable barotropic jet from a cumulant expansion. J. Atmos. Sci., 65, 1955-1966, doi:10.1175/2007JAS2510.1.

— W. Qi, and S. M. Tobias, 2015: Direct statistical simulation of a jet. Big Book of Jets, B. Galperin and P. Read, Eds., Cambridge University Press, in press.

Merlis, T. M., and T. Schneider, 2009: Scales of linear baroclinic instability and macroturbulence in dry atmospheres. J. Atmos. Sci., 66, 1821-1833, doi:10.1175/2008JAS2884.1.

Nakamura, N., and D. Zhu, 2010: Finite-amplitude wave activity and diffusive flux of potential vorticity in eddy-mean flow interaction. J. Atmos. Sci., 67, 2701-2716, doi:10.1175/2010JAS3432.1.

O'Gorman, P. A., 2011: The effective static stability experienced by eddies in a moist atmosphere. J. Atmos. Sci., 68, 75-90, doi:10.1175/2010JAS3537.1.

_ tistics in a general circulation model without nonlinear eddy-eddy interactions. Geophys. Res. Lett., 34, L22801, doi:10.1029/2007GL031779.

Potter, S. F., T. Spengler, and I. M. Held, 2013: Reflection of barotropic Rossby waves in sheared flow and validity of the WKB approximation. J. Atmos. Sci., 70, 2170-2178, doi:10.1175/ JAS-D-12-0315.1.

Randel, W., and I. Held, 1991: Phase speed spectra of transient eddy fluxes and critical layer absorption. J. Atmos. Sci., 48, 688-697, doi:10.1175/1520-0469(1991)048<0688: PSSOTE $>2.0 . \mathrm{CO} ; 2$.

Robinson, W. A., 1997: Dissipation dependence of the jet latitude. J. Climate, 10, 176-182, doi:10.1175/1520-0442(1997)010<0176: DDOTJL $>2.0 . \mathrm{CO} ; 2$.

Salyk, C., A. P. Ingersoll, J. Lorre, A. Vasavada, and A. D. Del Genio, 2006: Interaction between eddies and mean flow in Jupiter's atmosphere: Analysis of Cassini imaging data. Icarus, 185, 430-442, doi:10.1016/j.icarus.2006.08.007.

Schneider, T., 2004: The tropopause and the thermal stratification in the extratropics of a dry atmosphere. J. Atmos. Sci., 61, 1317-1340, doi:10.1175/1520-0469(2004)061<1317:TTATTS > 2.0.CO;2.

- 2006: The general circulation of the atmosphere. Annu. Rev. Earth Planet. Sci., 34, 655-688, doi:10.1146/ annurev.earth.34.031405.125144.

— roturbulence into critical states of weak nonlinear eddy-eddy interactions. J. Atmos. Sci., 63, 1569-1586, doi:10.1175/JAS3699.1. , and P. A. O'Gorman, 2008: Moist convection and the thermal stratification of the extratropical troposphere. J. Atmos. Sci., 65, 3571-3583, doi:10.1175/2008JAS2652.1.

, and C. C. Walker, 2008: Scaling laws and regime transitions of macroturbulence in dry atmospheres. J. Atmos. Sci., 65, 2153 2173, doi:10.1175/2007JAS2616.1.
- and J. Liu, 2009: Formation of jets and equatorial superrotation on Jupiter. J. Atmos. Sci., 66, 579-601, doi:10.1175/ 2008JAS2798.1.

Seager, R., N. Harnik, Y. Kushnir, W. Robinson, and J. Miller, 2003: Mechanisms of hemispherically symmetric climate variability. J. Climate, 16, 2960-2978, doi:10.1175/1520-0442(2003)016<2960: MOHSCV $>2.0 . \mathrm{CO} ; 2$.

Shepherd, T. G., 1987: A spectral view of nonlinear fluxes and stationary-transient interaction in the atmosphere. J. Atmos. Sci., 44, 1166-1179, doi:10.1175/1520-0469(1987)044<1166: ASVONF $>2.0 . \mathrm{CO} ; 2$.

Simmons, A. J., and B. J. Hoskins, 1978: The life cycles of some nonlinear baroclinic waves. J. Atmos. Sci., 35, 414-432, doi:10.1175/1520-0469(1978)035<0414:TLCOSN>2.0.CO;2.

—, and —, 1980: Barotropic influences on the growth and decay of nonlinear baroclinic waves. J. Atmos. Sci., 37, 1679-1684, doi:10.1175/1520-0469(1980)037<1679: BIOTGA $>2.0 . \mathrm{CO} ; 2$.

- and D. M. Burridge, 1981: An energy and angularmomentum conserving vertical finite-difference scheme and hybrid vertical coordinates. Mon. Wea. Rev., 109, 758-766, doi:10.1175/1520-0493(1981)109<0758:AEAAMC>2.0.CO;2.

Smagorinsky, J., S. Manabe, and J. L. Holloway Jr., 1965: Numerical results from a nine-level general circulation model of the atmosphere. Mon. Wea. Rev., 93, 727-768, doi:10.1175/ 1520-0493(1965)093<0727:NRFANL>2.3.CO;2.

Srinivasan, K., and W. R. Young, 2012: Zonostrophic instability. J. Atmos. Sci., 69, 1633-1656, doi:10.1175/JAS-D-11-0200.1.

Stewartson, K., 1977: The evolution of the critical layer of a Rossby wave. Geophys. Astrophys. Fluid Dyn., 9, 185-200, doi:10.1080/ 03091927708242326.

Stone, P. H., 1972: A simplified radiative-dynamical model for the static stability of rotating atmospheres. J. Atmos. Sci., 29, 405-418, doi:10.1175/1520-0469(1972)029<0405: ASRDMF $>2.0 . \mathrm{CO} ; 2$.

Thorncroft, C., B. J. Hoskins, and M. E. McIntyre, 1993: Two paradigms of baroclinic-wave life-cycle behaviour. Quart. J. Roy. Meteor. Soc., 119, 17-55, doi:10.1002/ qj.49711950903.

Tobias, S. M., K. Dagon, and J. B. Marston, 2011: Astrophysical fluid dynamics via direct statistical simulation. Astrophys. J., 727, 127, doi:10.1088/0004-637X/727/2/127.

Uppala, S. M., and Coauthors, 2005: The ERA-40 Re-Analysis. Quart. J. Roy. Meteor. Soc., 131, 2961-3012, doi:10.1256/ qj.04.176.

Vallis, G. K., 2006: Atmospheric and Oceanic Fluid Dynamics: Fundamentals and Large-Scale Circulation. Cambridge University Press, 745 pp.

Warn, T., and H. Warn, 1978: The evolution of a nonlinear critical level. Stud. Appl. Math., 59, 37-71.

Whitaker, J. S., and P. D. Sardeshmukh, 1998: A linear theory of extratropical synoptic eddy statistics. J. Atmos. Sci., 55, 237-258, doi:10.1175/1520-0469(1998)055<0237: ALTOES $>2.0 . \mathrm{CO} ; 2$.

Williams, P. D., 2011: The RAW filter: An improvement to the Robert-Asselin filter in semi-implicit integrations. Mon. Wea. Rev., 139, 1996-2007, doi:10.1175/2010MWR3601.1.

Zhang, Y., and I. M. Held, 1999: A linear stochastic model of a GCM's midlatitude storm tracks. J. Atmos. Sci., 56, 3416-3435, doi:10.1175/1520-0469(1999)056<3416: ALSMOA $>2.0 . \mathrm{CO} ; 2$. 